\title{
The synergistic Reduning and cefmetazole sodium treatment of severe pneumonia is mediated by the AhR-Src-STAT3 pathway
}

\author{
Shanjun Luo ${ }^{1 \#}$, Lianfang Gan ${ }^{2,3,4 \#}$, Shengxing Liu ${ }^{1}$, Lifan Zhong ${ }^{2,3,4}$, Meiling Chen ${ }^{1}$, Hong Zhang $^{1}$, \\ Jiankang $\mathrm{Li}^{1}$, Ling Huang ${ }^{2,3,4,5}$, Chuanzhu $\mathrm{Lv}^{1,2,6}$
}

${ }^{1}$ Key Laboratory of Emergency and Trauma, Ministry of Education, College of Emergency and Trauma, Hainan Medical University, Haikou, China; ${ }^{2}$ Research Unit of Island Emergency Medicine, Chinese Academy of Medical Sciences, Hainan Medical University, Haikou, China; ${ }^{3}$ Research Center for Drug Safety Evaluation of Hainan Province, Hainan Medical University, Haikou, China; ${ }^{4}$ Hainan Province Key Laboratory for Drug Preclinical Study of Pharmacology and Toxicology Research, Hainan Medical University, Haikou, China; ${ }^{5}$ State Key Laboratory of Trauma, Burns and Combined Injury, Department of Wound Infection and Drug, Daping Hospital, Army Medical University, Chongqing, China; ${ }^{6}$ Emergency Medicine Center, Sichuan Provincial People's Hospital, University of Electronic Science and Technology of China, Chengdu, China

Contributions: (I) Conception and design: L Huang, C Lv, S Luo, L Gan; (II) Administrative support: C Lv, S Luo; (III) Provision of study materials or patients: S Luo, L Gan; (IV) Collection and assembly of data: S Luo, L Gan, M Chen; (V) Data analysis and interpretation: S Luo, S Liu; (VI) Manuscript writing: All authors; (VII) Final approval of manuscript: All authors.

"These authors contributed equally to this work.

Correspondence to: Ling Huang. Research Unit of Island Emergency Medicine, Chinese Academy of Medical Sciences, Hainan Medical University, Haikou, China. Email: puer6@163.com; Chuanzhu Lv. Key Laboratory of Emergency and Trauma, Ministry of Education, College of Emergency and Trauma, Hainan Medical University, Haikou, China. Emergency Medicine Center, Sichuan Provincial People's Hospital, University of Electronic Science and Technology of China, Chengdu, China. Email: Lvchuanzhu677@126.com.

Background: Reduning (RDN) is a common Chinese medicine preparation with antibacterial, antiinflammatory, antiviral and immunomodulatory effects in respiratory infectious diseases. Clinically, it is used in combination with antibiotics, but its synergistic effect and mechanism in treating severe pneumonia remain unclear.

Methods: A rat model of severe pneumonia and an in vitro coculture model consisting of A549 and THP-1 cells were used to observe the synergistic effect of RDN on severe pneumonia. The inflammatory cytokines were tested by enzyme-linked immunosorbent assay (ELISA). The localization of Aryl hydrocarbon receptor (AhR) in A549 cells was observed by immunofluorescence, and the interaction of AhR and signal transducer and activator of transcription 3 (STAT3) proteins was observed by co-immunoprecipitation. AhR-Src tyrosine kinase (Src)-STAT3 pathway in rats and A549 cells were examined by Western Blot. Histopathological changes were observed by Hematoxylin-eosin (HE) staining, X-ray and survival rates were used to evaluate the effects of paclitaxel on severe pneumonia rats.

Results: RDN regulation of Src-STAT3-interleukin 10 (IL-10) signaling pathway activation and macrophage polarization were mediated through the nuclear receptor AhR. The expression of AhR was significantly increased after RDN treatment, and this effect was accompanied by STAT3 expression increasing. Coimmunoprecipitation confirmed an interaction between AhR and STAT3 and upregulated IL-10 expression. Silencing AhR decreased Src, STAT3, and IL-10 expression. RDN activated AhR and increased Src, STAT3, and IL-10 expression. In addition, RDN regulated the polarization of macrophages RDN combined with cefmetazole sodium significantly reduced the pulmonary bacterial load, alleviated lung injury, and reduced o inflammatory factors expression, improving their survival.

Conclusions: RDN can synergistically enhance the effect of cefmetazole sodium treatment in severe pneumonia, and the mechanism may involve increasing the expression level of IL-10 mediated through the AhR-Src-STAT3 pathway, driving the polarization of macrophages, and attenuating the cytokine storm to control inflammation in severe pneumonia. 
Keywords: Reduning; severe pneumonia; Aryl hydrocarbon receptor (AhR); signal transducer and activator of transcription 3 (STAT3); synergistic

Submitted Dec 27, 2021. Accepted for publication Feb 18, 2022.

doi: $10.21037 /$ jtd-22-126

View this article at: https://dx.doi.org/10.21037/jtd-22-126

\section{Introduction}

Severe pneumonia is an infectious pulmonary disease caused by pathogenic microbial infection, its severity is related to the body's immunity, viral-bacterial co-infection, the amount of infected bacteria, etc., and more importantly, it is closely related to the uncontrolled inflammatory response after pathogen infection (1-3). Currently, the mortality rate of patients with severe pneumonia is $25-50 \%$; thus, severe pneumonia is a serious challenge for intensive care unit (ICU) doctors (4). In the early stage of severe pneumonia, a large number of proinflammatory factors, such as tumor necrosis factor alpha (TNF- $\alpha$ ), interleukin (IL)-6, and IL-1 $\beta$, are produced, and the level of anti-inflammatory factor IL-10 is decreased, inducing a "cytokine storm." When not controlled in time, a cytokine storm can lead to serious pathophysiological events (5). Cefmetazole sodium (CMZ) is a broad-spectrum antibiotic commonly used in the clinical treatment of severe pneumonia, in which it plays a very important role in improving patient prognosis (6). The abuse of antibiotics and the emergence of drugresistant bacteria have become a difficult problem for ICU doctors (7). In addition to the application of effective antimicrobial agents, the clinical medication regimen for severe pneumonia often requires the treatment of the inflammatory response and immune regulation (8). Thus, finding key therapeutic targets and administering effective drugs in a timely manner to prevent cytokine storms in the early stage of severe pneumonia are key to determining the treatment success of severe pneumonia.

Aryl hydrocarbon receptor (AhR) is a member of the basic helix-loop-helix transcription factor superfamily. It is a ligand-activated transcription factor. AhR is indispensable in the regulation of the lipopolysaccharide (LPS)-induced inflammatory cascade, increased patient tolerance to LPS and protection against sepsis, and its expression level is closely related to the release of inflammatory factors (9). Previous study by our research group showed that upregulated AhR expression mediated an increase in IL-10 expression and downregulated IL-6 and IL-8 expression, thus inhibiting the inflammatory response, maintaining immune homeostasis, and regulating the occurrence and prognosis of sepsis (10). Signal transducer and activator of transcription 3 (STAT3) is a transcriptional regulatory cytokine that plays a key role in controlling inflammation and regulating immunity. The phosphorylation of key factors leads to physiological effects (11). AhR upregulates the phosphorylation levels of the Src tyrosine kinase (Src) and STAT3 to increase the expression of the antiinflammatory factor IL-10. The increased expression of IL-10 in the early stage of severe pneumonia can improve the body's tolerance to LPS, reducing the impact of the "early hyperinflammatory response" $(10,12)$. Additionally, the activation of AhR can regulate the polarization of macrophages (13), and M1 macrophages exert a great effect on lung injury (14). Thus, regulating the polarization direction of macrophages can help to control the inflammatory response and improve the therapeutic effect on severe pneumonia.

Reduning (RDN) is a type II traditional Chinese medicine (TCM) injection made from Artemisia annua, honeysuckle, and gardenia jasminoides, and it has been proven to have a wide range of pharmacological effects, including antibacterial, immunomodulatory, antiviral, and anti-inflammatory effects (15), Studies have found that its main active ingredients, genipin, geniposide, caffeic acid, and chlorogenic acid, have good anti-inflammatory effects $(16,17)$. RDN is often used in combination with antiviral drugs and antibiotics to treat respiratory tract infections. It is also listed in the 8th edition of China's Coronavirus Disease 2019 (COVID-19) Treatment Guidelines as a TCM preparation for the treatment of severe COVID-19 $(18,19)$, but its effect on severe pneumonia is still unclear. Thus, we investigated the effects of RDN on LPS-induced severe pneumonia models consisting of A549 cells, a coculture model consisting of A549 and THP-1 cells, and rats infected with Klebsiella pneumoniae, and explored its mechanism of action. Our findings are providing evidence for RDN synergistic antibiotics in the treatment of severe pneumonia. We present the following article in accordance with the ARRIVE reporting checklist (available at https:// jtd.amegroups.com/article/view/10.21037/jtd-22-126/rc). 


\section{Methods}

\section{Drugs and regents}

RDN $(2.6 \mathrm{~g} / \mathrm{mL})$ for use in injections was provided by Jiangsu Kanion Pharmaceutical Co., Ltd. (Jiangsu, China). CMZ was obtained from Sinopharm Zhijun Pharmaceutical Co., Ltd. (Shenzhen, China). LPS (Escherichia coli 055:B5) and Cell Counting Kit-8 (CCK-8) were purchased from Sigma Chemical Co. (St. Louis, MO, USA). IL-4, IL-13, and interferon gamma (IFN- $\gamma$ ) were purchased from PeproTech Inc. (Rocky Hill, NJ, USA). IL-1 $\beta$, IL-6, IL-10, and TNF- $\alpha$ enzyme-linked immunosorbent assay (ELISA) kits were obtained from Servicebio Biotech Co., Ltd. (Nanjing, China). Antibodies against STAT3, phosphorylated (p)-STAT3, Src, p-Src, and $\beta$-actin were purchased from Abcam (Cambridge, UK). Antibodies against AhR, IL-10, cluster of differentiation (CD)86, and CD163 were purchased from Abmart (Shanghai, China). Horseradish peroxidase (HRP)-conjugated goat anti-rabbit antibodies were provided by Biosharp (Hefei, China). A Pierce ${ }^{\mathrm{TM}}$ classic magnetic immunoprecipitation (IP)/ coimmunoprecipitation (co-IP) kit (88804) was purchased from Thermo Fisher Scientific (Waltham, MA, USA). AhR short hairpin ribonucleic acid (shRNA)-expressing lentivirus was obtained from GeneChem Co., Ltd. (Shanghai, China).

\section{Cell lines and culture}

A549 cells (Procell CL-0016) and THP-1 cells (Procell CL-0233) were kindly provided by Procell Life Science and Technology Co., Ltd. (Wuhan, China). The cells were cultured with Roswell Park Memorial Institute (RPMI)1640 medium containing $10 \%$ fetal bovine serum, $100 \mathrm{U} / \mathrm{mL}$ of penicillin, and streptomycin in an incubator at $37{ }^{\circ} \mathrm{C}$ and $5 \%$ carbon dioxide $\left(\mathrm{CO}_{2}\right)$.

The A549 and THP-1 cells were cocultured in $2 \mathrm{~mL}$ of 1,640 complete medium containing $100 \mathrm{ng} / \mathrm{mL}$ of Phorbol-12-myristate-13-acetate (PMA) in the upper layer of the coculture plate. Specifically, the THP-1 cells were seeded at a density of $1 \times 10^{6}$ cells/well in a 6 -well plate, and induced to differentiate into M0 macrophages for 48 hours. A medium containing $20 \mathrm{ng} / \mathrm{mL}$ of IFN- $\gamma$, $100 \mathrm{ng} / \mathrm{mL}$ of LPS or $20 \mathrm{ng} / \mathrm{mL}$ of IL-4, and $20 \mathrm{ng} / \mathrm{mL}$ of IL-13 was added to the M0 macrophages to induce their differentiation into M1 or M2 macrophages, and the polarized cells were then cocultured with the A549 cells seeded in the bottom layer.

\section{CCK-8 assay}

The A549 cells were seeded in 96-well culture plates, and the concentration was adjusted to $3 \times 10^{3}$ cells/well. After 8-12 hours, the cultured cells were treated with different concentrations of RDN (i.e., $0.1,0.2,0.4,0.8$, or $1.6 \mathrm{mg} / \mathrm{mL}$ ) for 2 hours, followed by stimulation with LPS $(20 \mu \mathrm{g} / \mathrm{mL})$ for 48 hours. All of the experimental groups were treated with CCK- 8 reagent $(10 \mu \mathrm{L} /$ well $)$ and incubated for 2 hours. The optical density was measured at $450 \mathrm{~nm}$ with a microplate reader.

\section{Lentiviral transfection and the selection of stably transfected cell lines}

A549 cells were seeded in $24-w e l l$ plates at $3 \times 10^{4}$ cells $/ \mathrm{mL}$ and cultured at $37^{\circ} \mathrm{C}$ with $5 \% \mathrm{CO}_{2}$ for 12 hours. The culture was maintained with medium containing lentivirus expressing shRNA against AhR. After 72 hours, fluorescence expression was observed with an inverted fluorescence microscope. Next, $2 \mu \mathrm{g} / \mathrm{mL}$ of puromycin solution was used for screening, and the medium containing puromycin was changed every 2 days for 14 days. AhR-treated stably shRNA-transfected A549 cell lines (AhR23 ${ }^{--}$and AhR2 $4^{--}$) were thus obtained. The messenger RNA (mRNA) and protein levels of AhR were verified by quantitative reverse transcription polymerase chain reaction (qRT-PCR) and Western blot analyses.

\section{ELISA}

The supernatant was collected from each group of treated cells, and serum was collected from the rats and stored at $-80{ }^{\circ} \mathrm{C}$. Next, the concentrations of IL- $1 \beta$, IL- 6 , TNF- $\alpha$, and IL-10 were assessed with enzyme-linked immunoassay (ELISA) kits. The absorbance was measured at $450 \mathrm{~nm}$.

\section{Co-IP}

The cells were collected and lysed, and the lysate was incubated for 30 minutes on ice, and then centrifuged at $12,000 \mathrm{r} / \mathrm{min}$ at $4{ }^{\circ} \mathrm{C}$ for 15 minutes. Total protein concentration was measured using the bicinchoninic acid (BCA) method. The antibody was added and incubated at $4{ }^{\circ} \mathrm{C}$ overnight. The protein $\mathrm{A} / \mathrm{G}$ beads were added and incubated in a shaker at $4^{\circ} \mathrm{C}$ for 2 hours. The antigenantibody complexes were eluted from the immunomagnetic beads, sample loading buffer was added, and the beads were 
Table 1 The primers were designed and synthesized by Sangon Biotech Co., Ltd.

\begin{tabular}{lcc}
\hline Gene & Forward primer & Reverse primer \\
\hline TNF- $\alpha$ & GGTAAGGGAAGAGGTCGGC & TTGCTTCTTCCCTGTTCCCC \\
IL-1 $\beta$ & CCAAAGAACAGGAAGCGGC & ATCTGGTGTCCTTGCTGCTC \\
IL-6 & AAGTGATGACGGCCCAATGT & CCAGACAGGGAAGGAACCAC \\
AhR & AGCTCACATCTTCACACGGG & GTCCCCTTAGTCTTGCGCAT \\
SrC & ATGTGGTGGGGATCTGAGGA & ACAAGAGGTCAGCTCAGCAC \\
STAT3 & GTTGGGGCAGTACATGGACA & GCCTTGCGCTTCCTAGGTAA \\
IL-10 & GAGTGCACGCAGGTACATCT & ACTGATGTTGGAGCTCTGCC \\
GADPH & TGATGGATGGCAGGGTTTCC & CCATCATGACTCAGCTCCCC \\
\hline
\end{tabular}

boiled for 5 minutes. Finally, Western blot analyses were performed for protein detection.

\section{QRT-PCR analysis}

Total RNA was extracted using an RNA extraction kit (Takara). One microgram of total RNA was synthesized into complementary deoxyribonucleic acid (DNA) by reverse transcription, and the mRNA expression level of the target gene was detected by qRT-PCR using a TB Green Premix kit (Takara). qRT-PCR was performed on a quantitative Studio3 7,500 real-time PCR system. Glyceraldehyde 3-phosphate dehydrogenase $(G A P D H)$ was selected as the reference gene. The sequences and names of the primers used in this experiment are listed below (Table 1).

\section{Western blot analyses}

Protein lysate was added to the cells or tissues and homogenized on ice for 30 minutes. The supernatant was centrifuged at $12,000 \mathrm{r} / \mathrm{min}$ at $4{ }^{\circ} \mathrm{C}$ for 15 minutes, and the protein concentration of the supernatant was determined with a BCA protein analysis kit. Protein samples $(20 \mu \mathrm{g}$ of each sample) were separated by sodium dodecyl sulfate polyacrylamide gel electrophoresis, and transferred to polyvinylidene fluoride membranes. Five percent skim milk was incubated with the membranes at room temperature for 2 hours, and 5\% BSA was used to block the phosphorylated proteins. Antibodies against AhR, p-Src, Src, p-STAT3, STAT3, $\beta$-Actin, IL-10, CD86, and CD163 were incubated with the membranes at $4{ }^{\circ} \mathrm{C}$ in a shaker overnight. Tris Buffer Solution-Tween20 (TBST) was used to wash the membranes 6 times for 5 minutes each time. The HRPlabeled secondary antibodies were incubated with the membrane at room temperature for 1.5 hours. TBST was used to wash the membranes 6 times for 5 minutes each time. Enhanced chemiluminescence was used for development, and gray strip values were determined with an ImageJ software image analysis system.

\section{Molecular docking analysis}

The SWISS-MODEL server was used to model the homologous protein structures. AutoDock Tools 1.5.6 software was used to delete water molecules from the target protein and to add nonpolar hydrogen to calculate the Gasteiger charge. The ligand structure was analyzed with chem3D software. AutoDock Tools 1.5.6 software was imported, the atomic charge was added, the atomic type was specified, and all key flexible components in the default docking ligands were rotated. AutoDock Vina 1.1.2 software was used for docking, and Discovery Studio 2016 software was used to visualize the docking results.

\section{Animal model preparation and administration}

Male Sprague-Dawley (SD) rats weighing approximately 180-220 g were purchased from Hunan SJA Laboratory Animal Co., Ltd. under production license No. SCXK (Hunan) 2019-0004. AK pneumoniae strain was inoculated into Luria-Bertani (LB) solid medium and cultured in a $\mathrm{CO}_{2}$ incubator at $37^{\circ} \mathrm{C}$ for 16 hours before the experiment. The bacteria were collected and diluted with normal saline. The $\mathrm{McF}$ arland value was measured on a turbidimeter and adjusted to $160 \mathrm{McF}$ arland $\left(4.8 \times 10^{10}\right.$ colony-forming unit $\left./ \mathrm{mL}\right)$. After successful anesthesia with pentobarbital sodium $(50 \mathrm{mg} / \mathrm{kg}), 0.35 \mathrm{~mL}$ of the bacterial solution was injected into the trachea of each rat, and the establishment of the 
model was confirmed 4 days later.

The rats were assigned to 10 groups: (a) the normal control group; (b) the sham operation group (airway infused with normal saline), (c) the model group; (d-f) the RDN (2.7, 5.4, and $10.8 \mathrm{~g} / \mathrm{kg})$ groups; (g) the CMZ (0.42 g/kg) group; and $(\mathrm{h}, \mathrm{i}, \mathrm{n})$ : the CMZ $(0.42 \mathrm{~g} / \mathrm{kg})+\mathrm{RDN}(2.7,5.4$, and $10.8 \mathrm{~g} / \mathrm{kg}$ ) groups. After 3 days of caudal vein administration of the treatment, pentobarbital sodium $(50 \mathrm{mg} / \mathrm{kg})$ was used to anesthetize the rats, and abdominal aorta blood was then taken, and the rats were sacrificed. All experiments were reviewed and approved by the Institutional Animal Care and Treatment Committee of Hainan Medical University (No. HYLL-2021-159), in compliance with Guidelines for the Humane Treatment of Laboratory Animals (Ministry of Science and Technology of the People's Republic of China; policy No. 2006398).

\section{Histopathological changes in the lung tissues}

The lung tissues were fixed with $4 \%$ paraformaldehyde for 24 hours, dehydrated and paraffin embedded. Paraffin slices of the lung tissue (4- $\mu \mathrm{m}$ thick) were prepared, and hematoxylin and eosin (H\&E) staining was used to observe the pathological changes in the lung tissues of the rats under a light microscope.

\section{Immunofluorescence technic}

The cells were immersed in phosphate buffered solution (PBS) 5 times, fixed with 4\% paraformaldehyde for 10 minutes, permeated with $0.3 \%$ Triton X-100 for 10 minutes, blocked with BSA for 1 hour, incubated with a specific primary antibody at $4{ }^{\circ} \mathrm{C}$ overnight, washed with PBS 5 times, incubated with the appropriate fluorescent secondary antibody, stained with 4',6-Diamidino-2phenylindole dihydrochloride (DAPI), and examined by laser confocal microscopy. The paraffin sections of lung tissue were dewaxed, antigen retrieval was performed, BSA was added for 30 minutes, and the primary antibodies (i.e., anti-AhR, anti-p-Src, anti-p-STAT3, anti-IL-10, antiCD86, and anti-CD163) were added and incubated at $4^{\circ} \mathrm{C}$ overnight. PBS was used to wash each blot 3 times for 5 minutes each time, and 2 fluorescent tags (Cy3-conjugated AffiniPure goat anti-rat IgG) were incubated for 1 hour at room temperature. DAPI was used to re-stain the nuclei, and the cells were incubated at room temperature in the dark for 10 minutes. The cells were then washed with PBS 3 times for 5 minutes each time, and the tablets were sealed with anti-fluorescence quenching reagent. Fluorescence microscopy was used to take pictures, and the absorbance value of the positive fluorescence signal was determined by ImageJ software and then analyzed.

\section{CXRs}

After the rats were anesthetized with pentobarbital sodium (50 mg/kg), chest X-rays (CXRs) were taken with a highresolution $\mathrm{X}$-ray machine, and images were collected.

\section{Survival analysis}

As mentioned above, the rats in each group were observed every 12 hours after treatment, and the number of deaths within 5 days was recorded for use in a survival analysis, and the mortality rate during the observation period was calculated.

\section{Colony-forming units of BALF}

After the rats were anesthetized with pentobarbital sodium (50 $\mathrm{mg} / \mathrm{kg}$ ), the left lung with the trachea was removed, the right bronchus was ligated, and $5 \mathrm{~mL}$ of normal saline at $4{ }^{\circ} \mathrm{C}$ was injected into the trachea for the lavage of the left lung. The operation was repeated 3 times to obtain bronchoalveolar lavage fluid (BALF). One milliliter of BALF was added to $9 \mathrm{~mL}$ of normal saline and diluted 106 times according to the method described above. The diluted liquid was inoculated into $\mathrm{LB}$ a solid medium, and cultured at $37^{\circ} \mathrm{C}$ for 24 hours. The colony-forming units were examined.

\section{Statistical analysis}

The statistical analysis and mapping were performed using GraphPad Prism 8.3 software. Kaplan-Meier survival curves were used to analyze the survival rate of each group. The experimental results are expressed as the mean \pm standard deviation (mean $\pm \mathrm{SD}$ ). Differences between groups were analyzed using a one-way analysis of variance and the Student's $t$-test. The results were considered statistically significant when the $\mathrm{P}$ value was $<0.05$ or $<0.01$.

\section{Results}

\section{In vitro study}

AhR can be combined with the main active components of RDN

In this study, the main active ingredients of RDN, including 
genipin, geniposide, caffeic acid, and chlorogenic acid, were selected to perform molecular docking with the target, AhR. The results showed that the active components formed hydrogen bonds with AhR, and that RDN could thus combine with AhR. The results suggested that AhR may be a target of RDN (see Figure $1 A$ ).

\section{Cytotoxicity evaluation of RDN in A549 cells}

The A549 cells were treated with RDN for 48 hours with or without LPS, and the effect of RDN on A549 cell viability was determined by CCK- 8 assays. The results showed that RDN at $0.1-0.8 \mathrm{mg} / \mathrm{mL}$ had no significant effect on the cell survival rate (see Figure $1 B$ ).

\section{RDN activates the Src-STAT3-IL-10 pathway mediated by $\mathrm{AhR}$ and inhibits the secretion of inflammatory factors}

As the results of the molecular docking experiments suggested that AhR might be a target of RDN, we measured AhR and downstream Src, STAT3, and IL-10 levels in RDN-treated A549 cells. As Figure $2 A$ shows, AhR expression increased after RDN treatment. Co-IP revealed a protein interaction between AhR and STAT3 (see Figure 2B). The expression of p-Src, P-STAT3, and IL-10 increased after AhR increased due to RDN treatment (see Figure 2C). The qRT-PCR results showed that RDN treatment led to the decreased expression of the proinflammatory factors of TNF- $\alpha$, IL-1 $\beta$, and IL-6, and the increased expression of the anti-inflammatory factor of IL-10. The levels of secreted inflammatory factors were detected by ELISAs, and the effect of RDN on the secretion of the inflammatory factors was observed. After RDN treatment, the levels of the proinflammatory factors decreased as expected, while those of the anti-inflammatory factors increased (see Figure 2D). In conclusion, RDN activated the Src-STAT3-IL-10 pathway through AhR, and inhibited the secretion of proinflammatory factors.

\section{AhR silencing inhibits Src-STAT3-IL-10 signaling pathway activation and increases the secretion of inflammatory factors}

We silenced $A b R$ loci 23 and 24 in A549 cells by transfection with $A b R$ shRNA lentivirus. $2 A b R$-treated stably shRNA-transfected A549 cell lines (i.e., $A b R 23^{-/-}$ and $A b R 24^{-}$) were obtained and verified by qRT-PCR and Western blot analyses (see Figure 3A). After $A b R$ silencing, the protein expression levels of p-Src, P-STAT3, and IL-10 decreased significantly. Conversely, after RDN was administered, the levels of p-Src, P-STAT3, and IL10 increased, but no significant difference was detected (see Figure $3 B$ ). The expression of proinflammatory factors increased and anti-inflammatory factors decreased after $A b R$ silencing. The levels of proinflammatory factors decreased and those of anti-inflammatory factors increased after RDN administration; however, no significant difference was detected (see Figure 3C). In summary, $A b R$ may be the main target of RDN.

\section{RDN Modulates M1/M2 polarization in the coculture system and activates the Src-STAT3-IL-10 signaling pathway through AhR}

We established a coculture model of A549 and THP-1 cells to explore the effects of RDN on macrophage polarization and the AhR-Src-STAT3-IL-10 pathway. CD86 is a marker of M1 macrophages, and CD163 is a marker of M2 macrophages. Western blotting also showed that RDN inhibited M1 expression and increased M2 expression (see Figure $4 A$ ). In cocultured cells in an inflammatory state, RDN also increased the expression of AhR in the nucleus, thus increasing the expression of p-Src, p-STAT3, and IL10 (see Figure 4B). However, in the coculture containing A549 and THP-1 cells in which AhR was silenced, the expression of p-Src, p-STAT3 and IL-10 was decreased (see Figure $4 C$ ). The results showed that RDN regulated M1/M2 polarization and activated the Src-STAT3-IL-10 signaling pathway through AhR in the coculture system.

\section{In vivo study}

RDN-enhanced CMZ reduces lung injury and increases the survival rate of rats with severe pneumonia

To explore the therapeutic effect of RDN and its combination with $\mathrm{CMZ}$ in rats with severe pneumonia, we observed the 5 -day survival of 10 groups of rats (15 per group). We found that $\mathrm{RDN}$ reduced the mortality of the rats with severe pneumonia, while CMZ combined with RDN significantly reduced rat mortality. RDN treatment also led to a reduction in the amount of bacteria in BALF. Additionally, the combined use of $C M Z$ and RDN had the most obvious effect on reducing the bacterial quantity in BALF (see Figure $5 A$ ). The CXRs showed that RDN reduced inflammatory exudate in the lungs, and RDN combined with CMZ most significantly reduced inflammatory exudate in the chest (see Figure $5 B$ ). Next, H\&E staining was used to observe the pathological changes in the lung tissues of rats with severe pneumonia. The lung tissues of the model group showed 
A

a

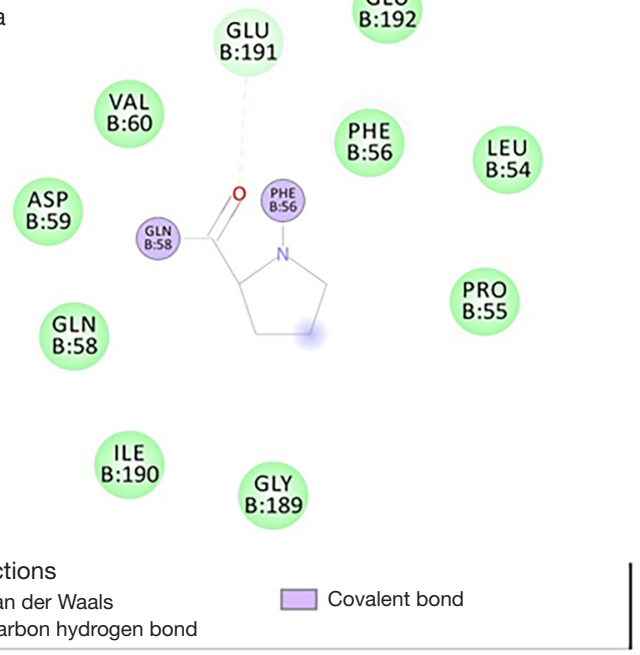

C

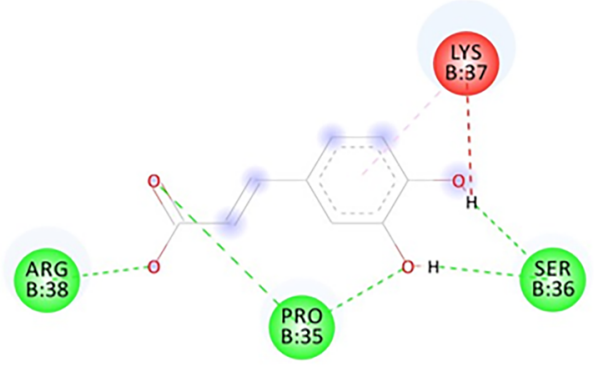

Interactions

$\square$ Conventional hydrogen bond $\quad \square$ Pi-Alkyl

Unfavorable Donor-Donor

B

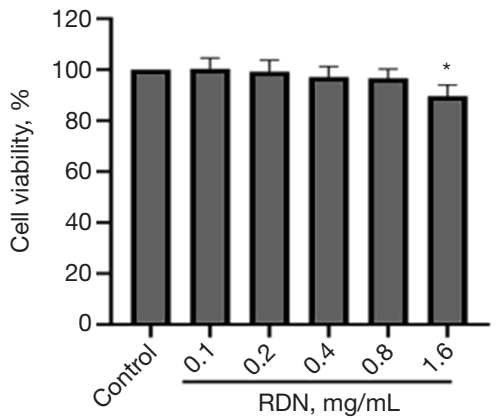

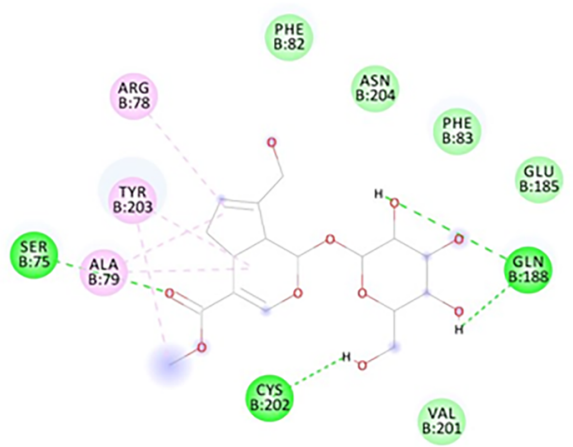

Interactions

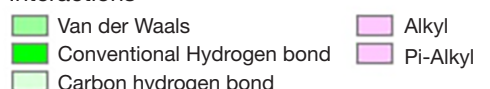

Carbon hydrogen bond

d
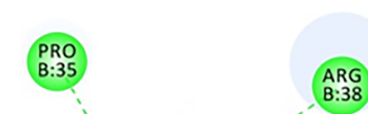

ARG
$B: 38$

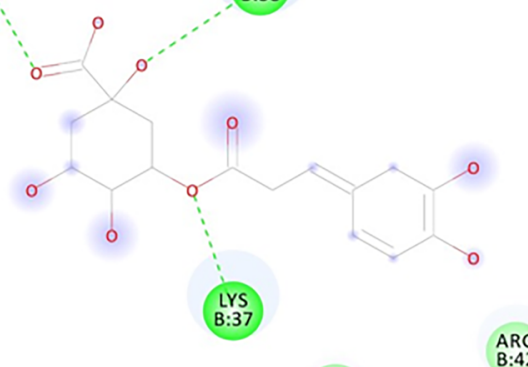

ASP
$B: 41$

Interactions

$\square$ Van der Waals

Conventional Hydrogen bond

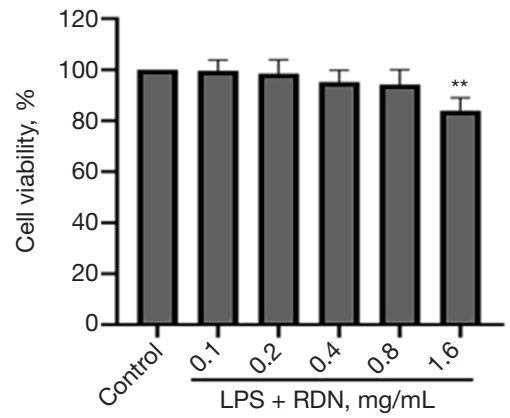

Figure 1 Molecular docking of RDN components with AhR. (A) AhR forms hydrogen bond bonds with genipin (a), geniposide (b), caffeic acid (c), and chlorogenic acid (d). Effects of RDN on the viability of A549 cells. (B) The cells were cultured with RDN (0.1, 0.2, 0.4, 0.8, or $1.6 \mathrm{mg} / \mathrm{mL})$ in the absence or presence of LPS $(20 \mu \mathrm{g} / \mathrm{mL})$ for $48 \mathrm{~h}$. The data are presented as the means $\pm \mathrm{SD}$. *, $\mathrm{P}<0.05,{ }^{* *}, \mathrm{P}<0.01 v s$. the control. RDN, Reduning; AhR, Aryl hydrocarbon receptor. 
A

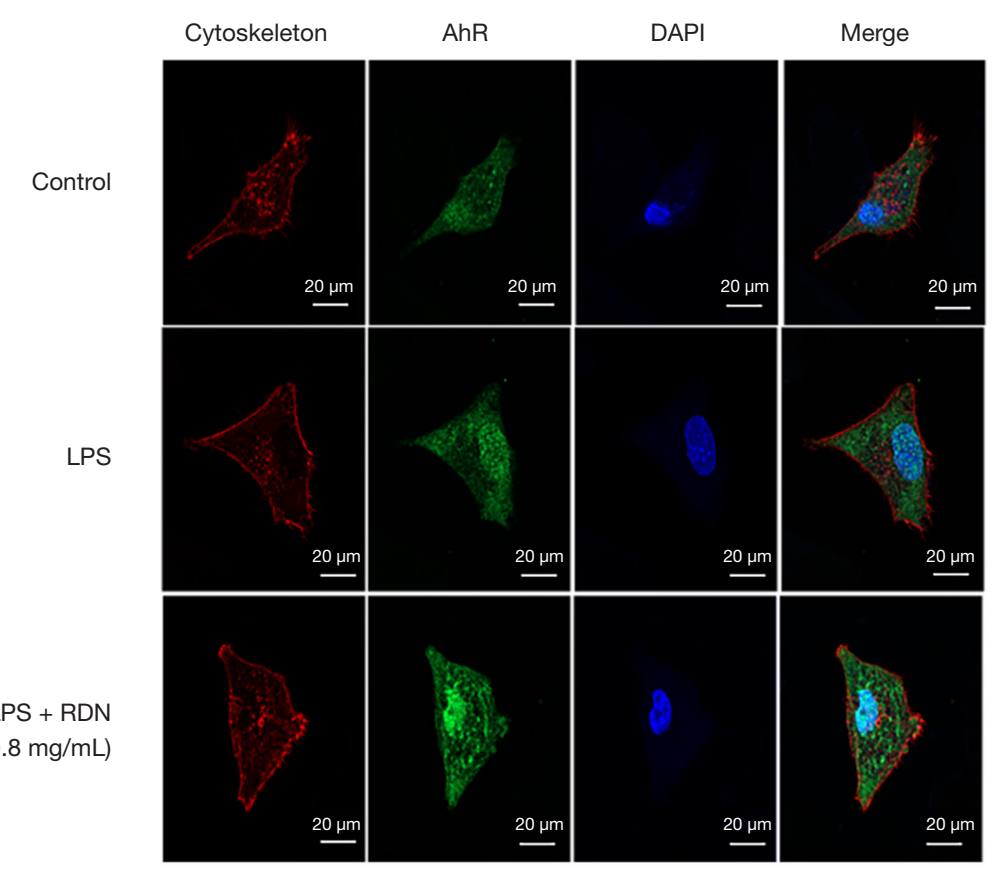

C
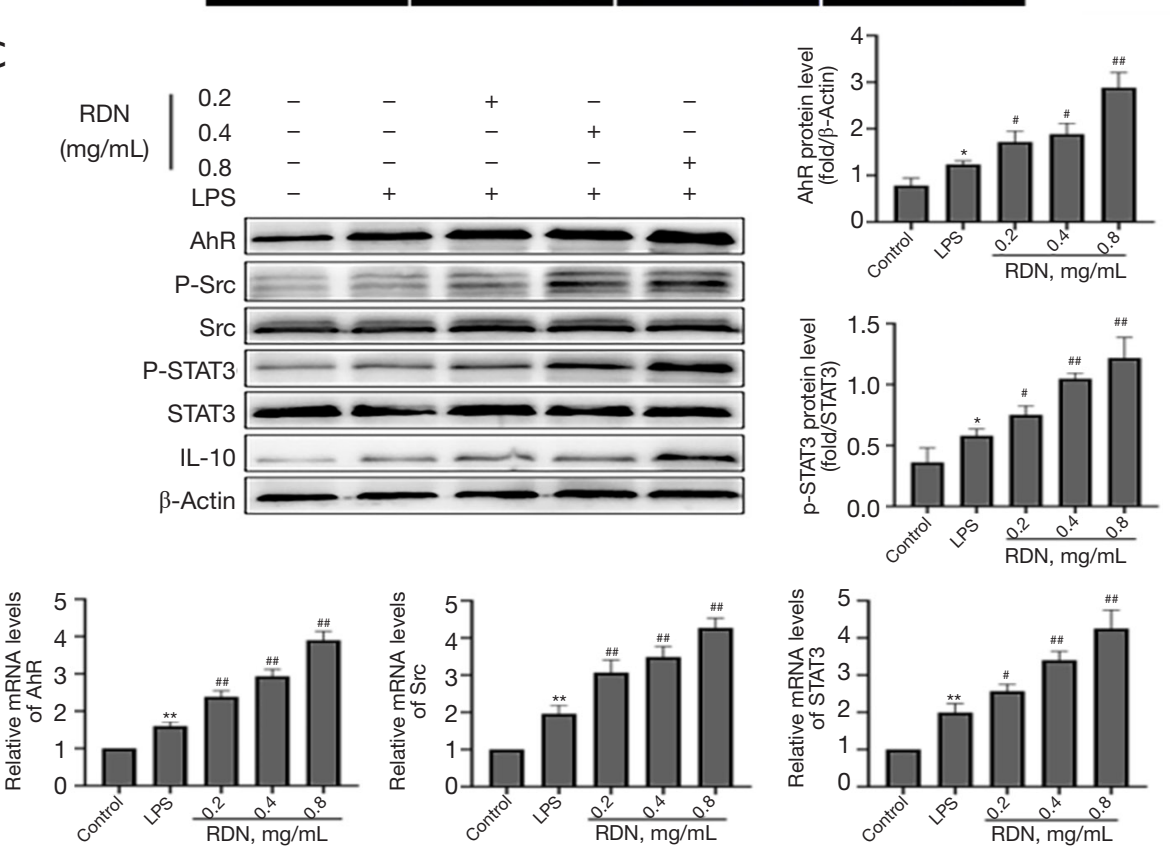

D
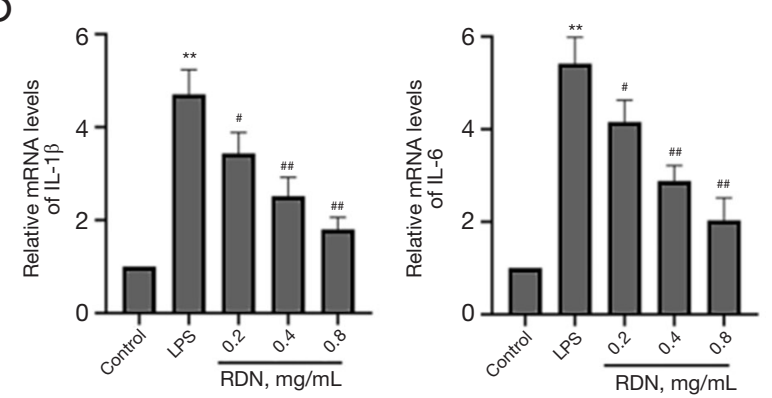

B
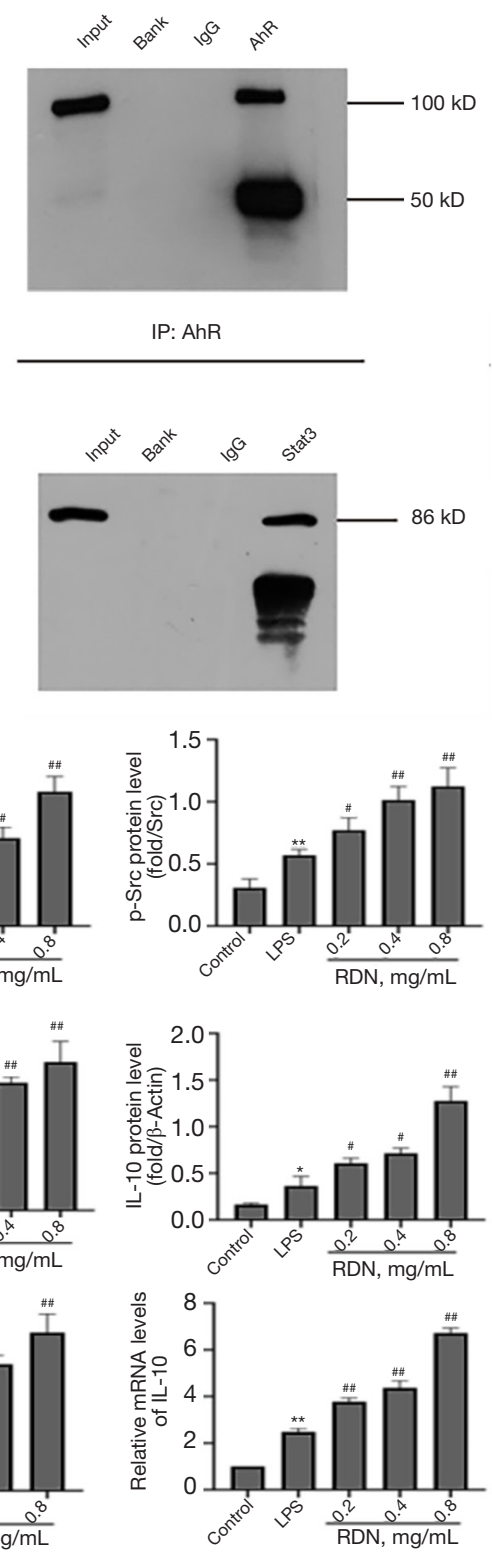

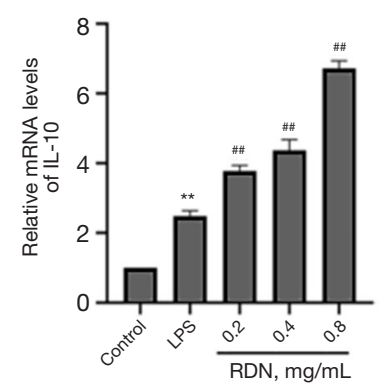



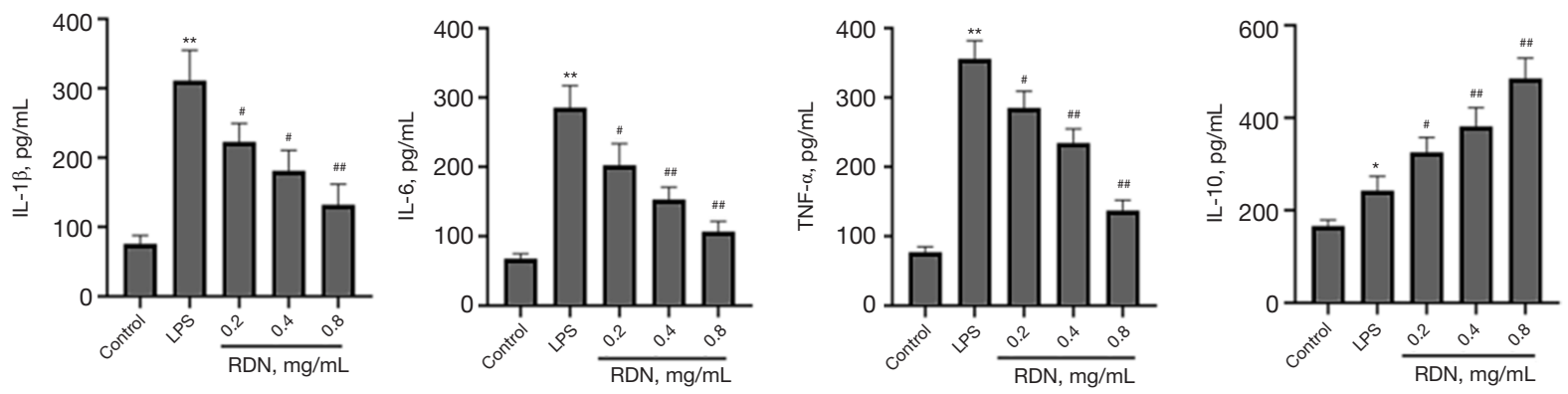

Figure 2 RDN activates the Src-STAT3-IL-10 pathway through AhR. (A) Immunofluorescence staining shows the expression of AhR (green) in A549 cells (×630). (B) The interaction between AhR and STAT3 as determined by co-IP. (C) Western blots showing AhR, p-Src, Src, p-STAT3, STAT3, IL-10, and $\beta$-actin expression, and qRT-PCR assay results showing AhR, Src, STAT3, and IL-10 expression in A549 cells. (D) qRT-PCR assay results showing IL-1 $\beta$, IL-6, TNF- $\alpha$, and IL-10 expression in A549 cells, and ELISA results showing IL-1 $\beta$, IL-6, TNF- $\alpha$, and IL-10 concentrations in the cell supernatant. The values represent the mean \pm SD of 3 independent experiments, and the differences between the mean values were assessed by the Student's $t$-test. * $, \mathrm{P}<0.05,{ }^{* *}, \mathrm{P}<0.01$ vs. the control group; ${ }^{*}, \mathrm{P}<0.05$, \#\#, $\mathrm{P}<0.01$ vs. the LPS group. RDN, Reduning; AhR, Aryl hydrocarbon receptor; p-Src, Phosphorylation Src tyrosine kinase; Src, Src tyrosine kinase; p-STAT3, Phosphorylation signal transducer and activator of transcription 3; STAT3, signal transducer and activator of transcription 3; IL-10, interleukin 10.

hyperplasia and consolidation, alveolar wall thickening, and lung hyperemia. There was massive hemorrhaging and a large population of neutrophils in the alveolar space, and fibrinous exudate in the alveolar space. These changes were alleviated after treatment with RDN and CMZ, and the greatest effect was evident in the RDN combined with CMZ group (see Figure 5C). The results showed that RDN combined with $\mathrm{CMZ}$ significantly reduced lung injury and increased the survival rate of rats with severe pneumonia.

\section{RDN reduces the expression of proinflammatory factors} and inflammatory indicators in rats with severe pneumonia and increases the production of anti-inflammatory factors Inflammatory factors (i.e., TNF- $\alpha$, IL- $1 \beta$, IL-6, and IL-10) and inflammatory markers (i.e., CRP and PCT) were detected using ELISA kits. As Figure 6 shows, RDN reduced the production of proinflammatory factors, reduced the level of inflammatory indicators, and increased the production of anti-inflammatory factors, and RDN combined with $\mathrm{CMZ}$ had the greatest effect on reducing proinflammatory factors, reducing inflammatory indicators, and increasing the production of anti-inflammatory factors. The results showed that RDN attenuated the inflammatory state of the rats with severe pneumonia.

\section{RDN activates Src-STAT3-IL-10 pathway through AhR} in synergistic treatment of severe pneumonia

To further explore the protective mechanism of RDN in severe pneumonia, we analyzed the expression of AhR, p-Src, p-STAT3, and IL-10 in lung tissues by immunofluorescence and Western blot analyses. The immunofluorescence results showed that the expression of AhR and its downstream proteins p-Src, p-STAT3, and IL-10 increased significantly after RDN treatment. However, after RDN was combined with CMZ treatment, the expression of these proteins increased even more significantly (see Figure $7 A$ ). These results were verified by Western blotting (see Figure $7 B$ ). The results showed that Src-STAT3 was activated by AhR in RDN-exposed tissues, IL-10 expression was increased, and the CMZ treatment of severe pneumonia was synergistically enhanced. The results showed that RDN activated Src-STAT3 through $\mathrm{AhR}$, increased IL-10 expression, exerted anti-inflammatory effects, and enhanced CMZ effects in the treatment of severe pneumonia.

\section{RDN suppresses M1 activation and promotes M2 polarization in rats with severe pneumonia}

To investigate the effect of RDN and its effect when combined with $\mathrm{CMZ}$ on macrophage polarization in rats with severe pneumonia, the levels of CD86, a marker of M1 macrophages, and CD163, a marker of M2 macrophages, were detected by immunofluorescence and Western blotting. The results showed that RDN inhibited M1 polarization and promoted M2 polarization, and the effect was more obvious after rats received the combination 
A
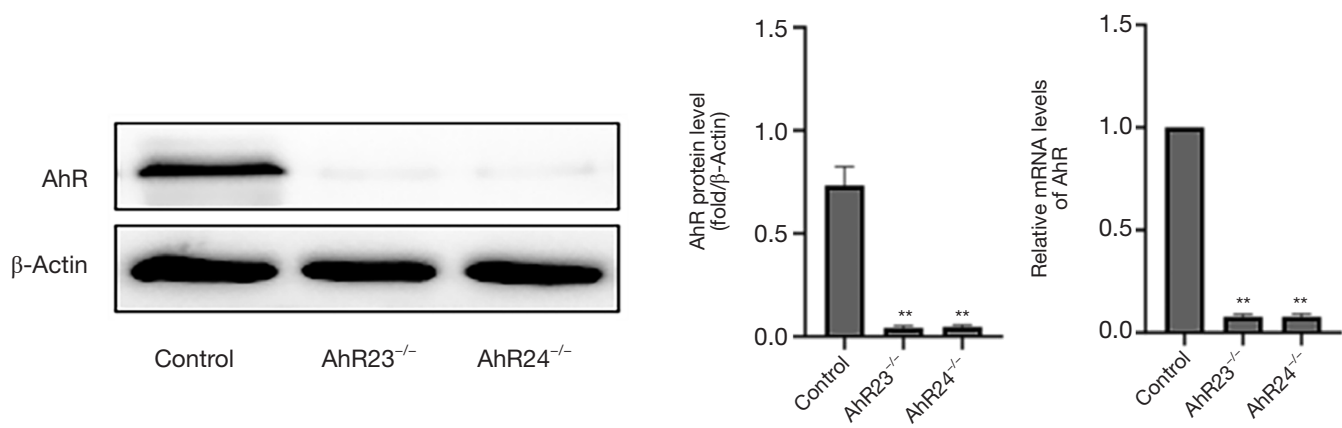

B
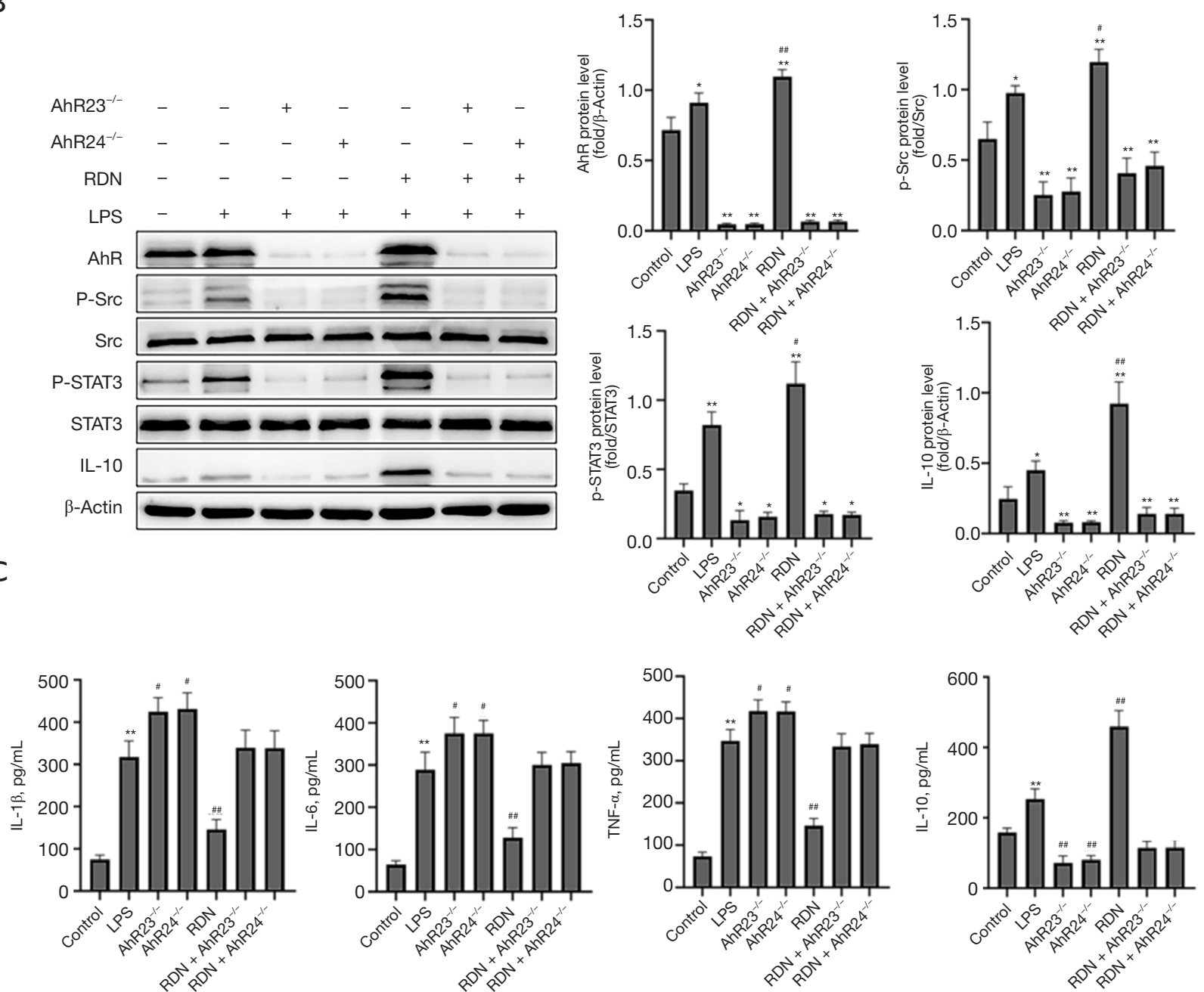

Figure 3 The effects of AhR silencing on the Src-STAT3-IL-10 pathway and inflammatory factors in A549 cells. (A) Detection of AhR expression after AhR shRNA lentivirus transfection of A549 cells. (B) Western blots showing AhR, p-Src, Src, p-STAT3, STAT3, IL-10, and $\beta$-actin levels in A549 cells. (C) ELISA showing the IL-1 $\beta$, IL-6, TNF- $\alpha$, and IL-10 concentrations in the cell supernatant. The values represent the mean \pm SD of 3 independent experiments, and the differences between the mean values were assessed by the Student's $t$-test. *, $\mathrm{P}<0.05$, **, $\mathrm{P}<0.01$ vs. the control group; ${ }^{\#}, \mathrm{P}<0.05$, ${ }^{\#}, \mathrm{P}<0.01$ vs. the LPS group. AhR, Aryl hydrocarbon receptor; $\mathrm{p}-\mathrm{Src}, \mathrm{Phosphorylation}$ Src tyrosine kinase; Src, Src tyrosine kinase; p-STAT3, Phosphorylation signal transducer and activator of transcription 3; STAT3, signal transducer and activator of transcription 3; IL-10, interleukin 10; LPS, lipopolysaccharide; ELISA, enzyme-linked immunosorbent assay; IL-6, interleukin 6; TNF- $\alpha$, tumor necrosis factor- $\alpha$. 
A

B
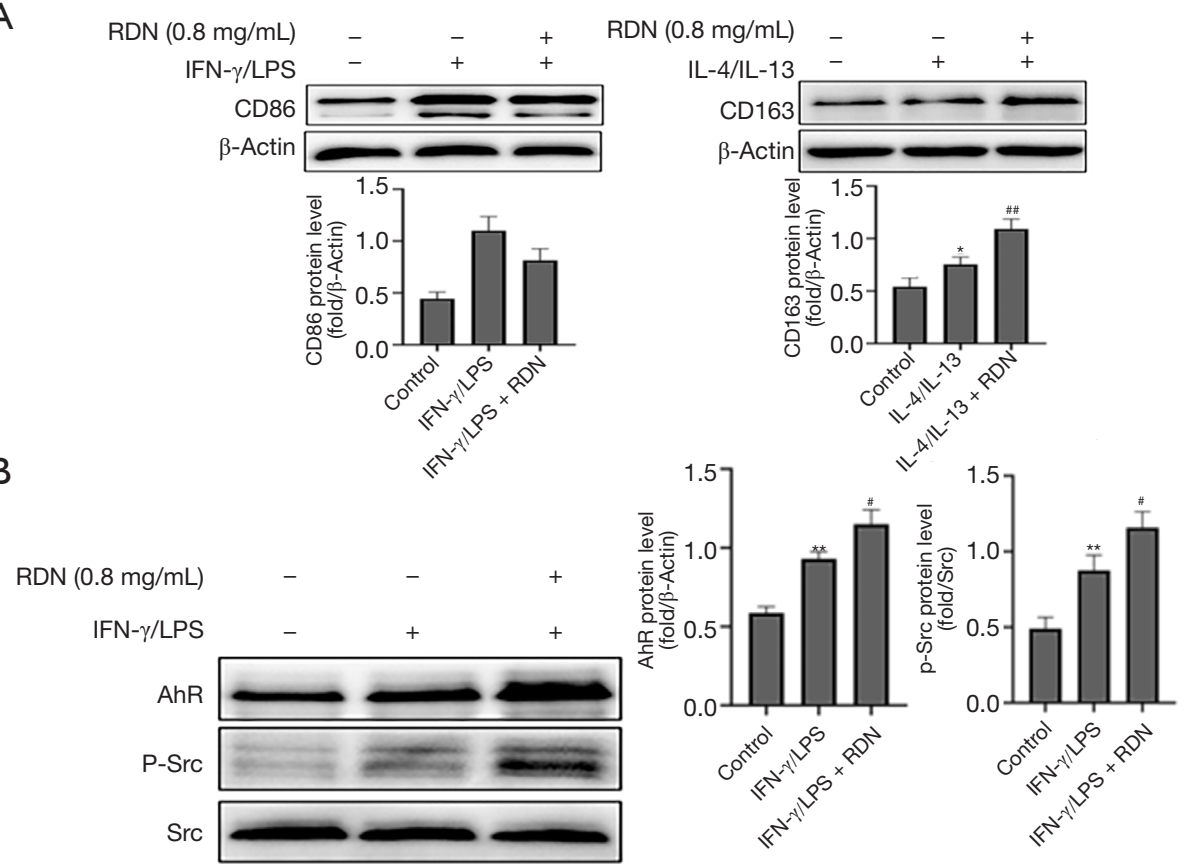

$\operatorname{RDN}(0.8 \mathrm{mg} / \mathrm{mL})$
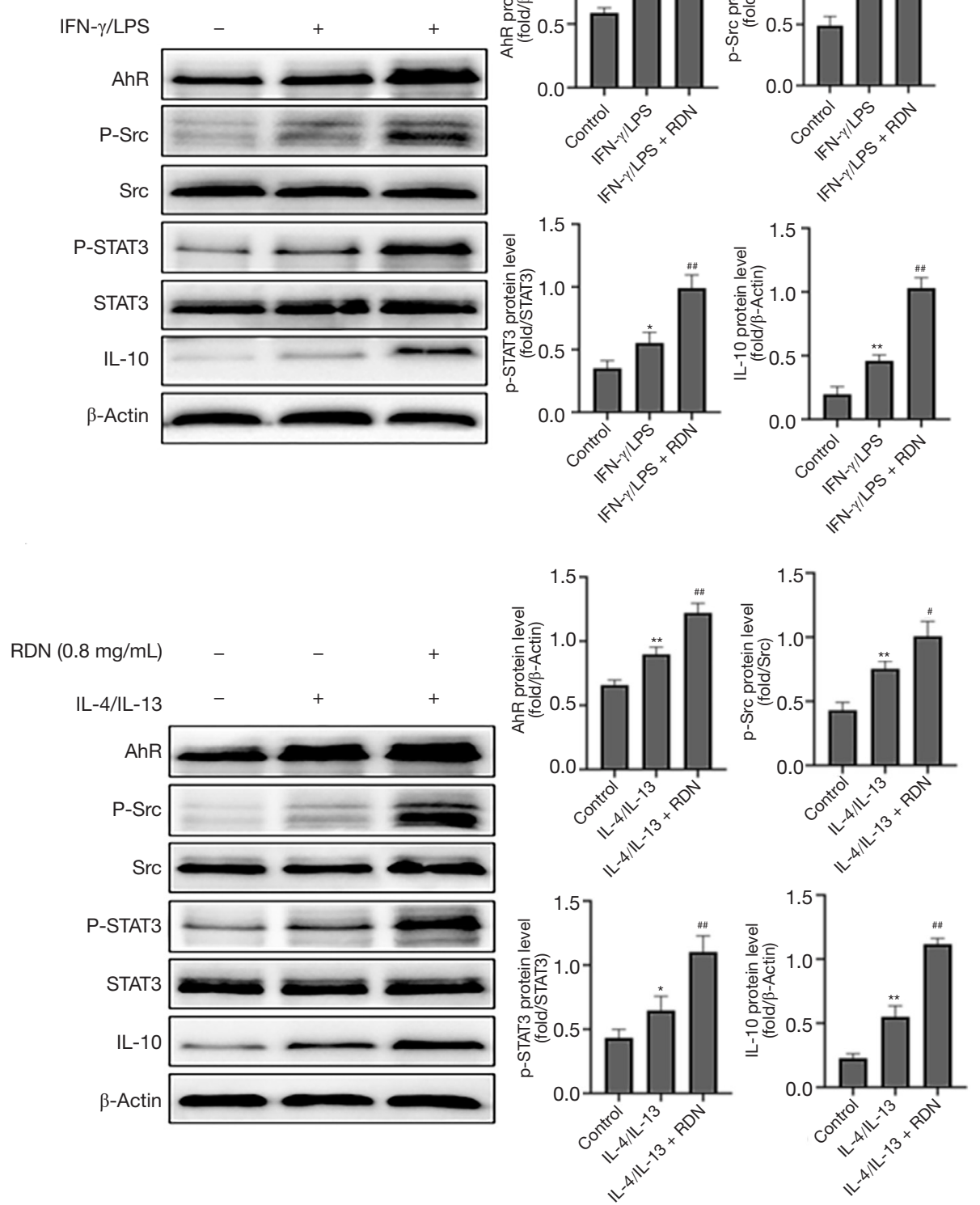

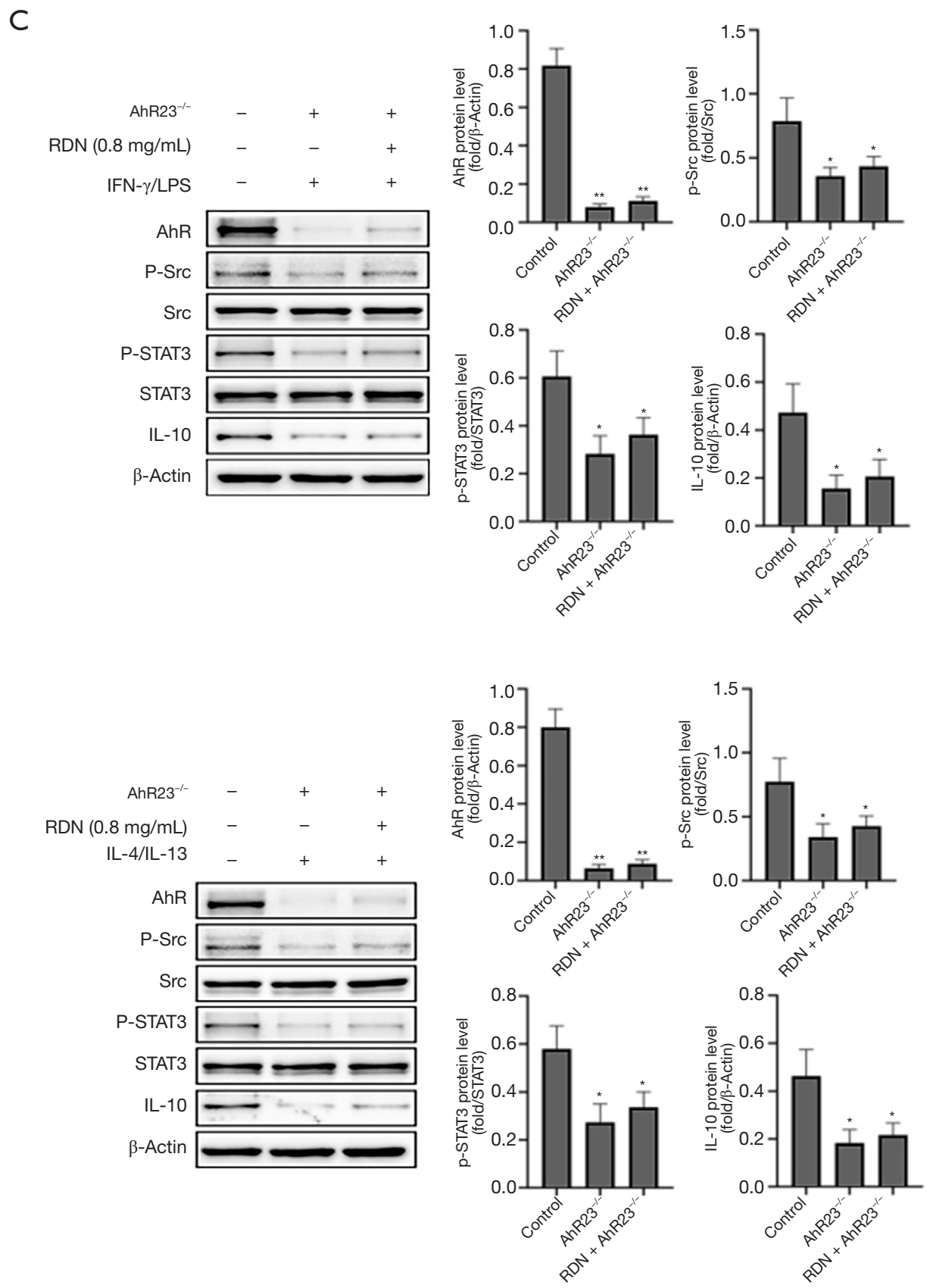

Figure 4 The effects of RDN on macrophage polarization and the A549 cell AhR-Src-STAT3-IL-10 pathway in the coculture system. (A) Western blot assay of CD86, CD163, and $\beta$-actin levels in THP-1 cells. (B,C) Western blot assay of AhR, p-Src, Src, p-STAT3, STAT3, IL-10, and $\beta$-actin levels in A549 cells and sh-AhR A549 cells. The values represent the mean \pm SD of 3 independent experiments, and the differences between the mean values were assessed by the Student's $t$-test. ${ }^{*}, \mathrm{P}<0.05,{ }^{* *}, \mathrm{P}<0.01$ vs. the control group; ${ }^{*}, \mathrm{P}<0.05$, ${ }^{\#}, \mathrm{P}<0.01$ $v s$. the IFN- $\gamma$, LPS, IL-4, and IL-13 groups. RDN, Reduning; AhR, Aryl hydrocarbon receptor; p-Src, Phosphorylation Src tyrosine kinase; Src, Src tyrosine kinase; p-STAT3, Phosphorylation signal transducer and activator of transcription 3; STAT3, signal transducer and activator of transcription 3; IL-10, interleukin 10; LPS, lipopolysaccharide; IL-4, interleukin 4; IL-13, interleukin 13; IFN- $\gamma$, interferon $\gamma$; CD86, cluster of differentiation 86; CD163, cluster of differentiation 163. 
A
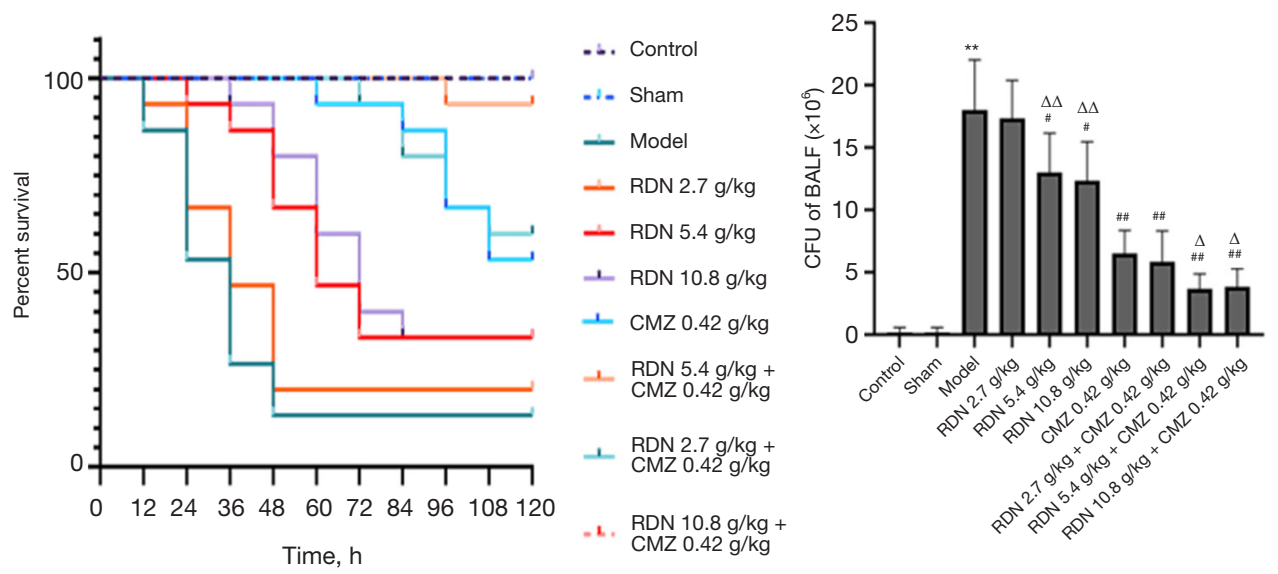

B
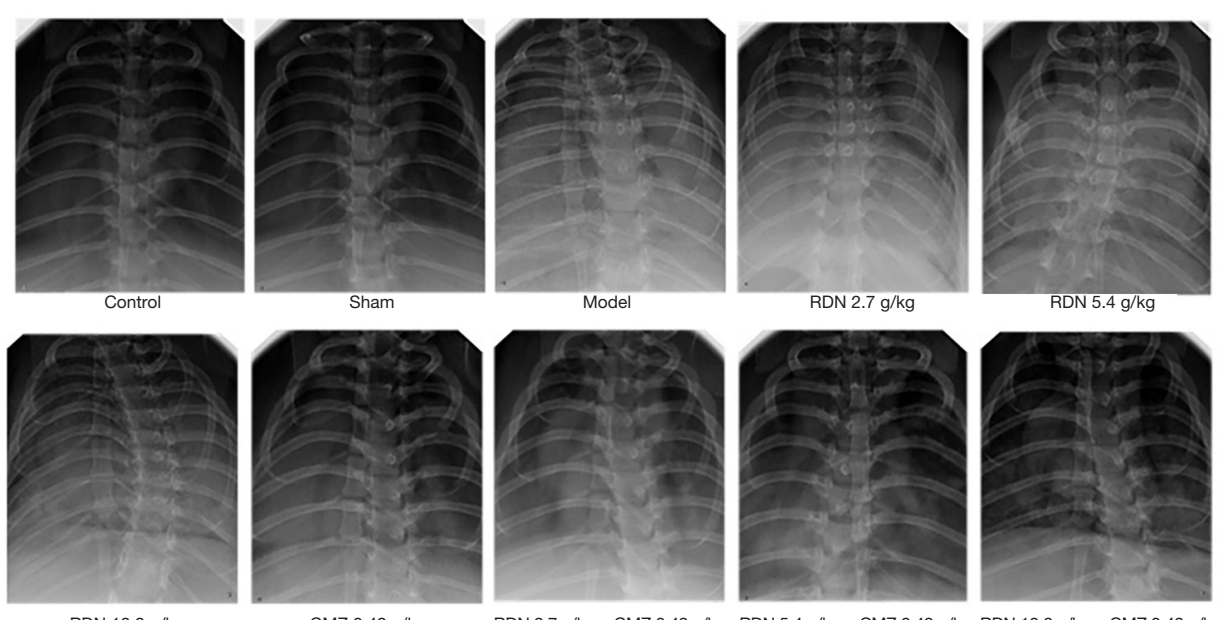

C
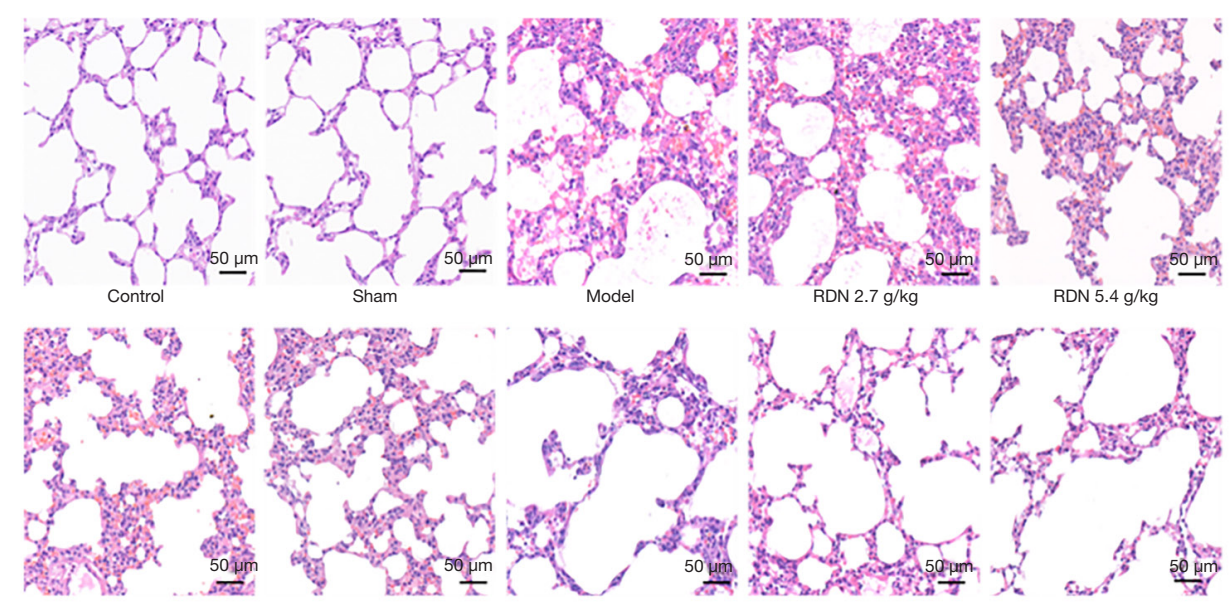

RDN $10.8 \mathrm{~g} / \mathrm{kg}$
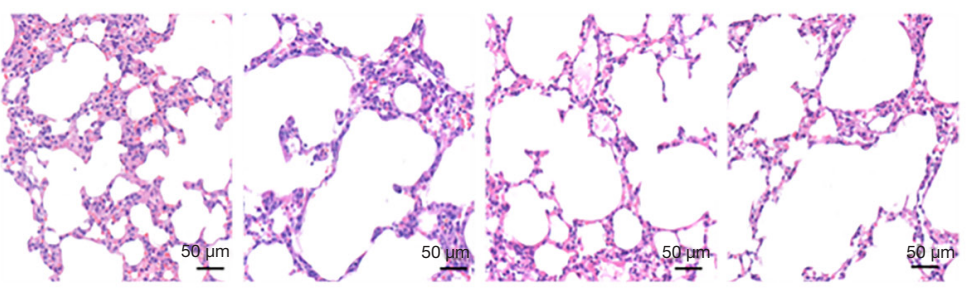

CMZ $0.42 \mathrm{~g} / \mathrm{kg}$

RDN $2.7 \mathrm{~g} / \mathrm{kg}+\mathrm{CMZ} 0.42 \mathrm{~g} / \mathrm{kg} \quad$ RDN $5.4 \mathrm{~g} / \mathrm{kg}+\mathrm{CMZ} 0.42 \mathrm{~g} / \mathrm{kg}$ RDN $10.8 \mathrm{~g} / \mathrm{kg}+\mathrm{CMZ} 0.42 \mathrm{~g} / \mathrm{kg}$

Figure 5 Effects of RDN on survival rate, lung histopathological changes and X-ray in severe pneumonia rats. (A) Survival rate and bacterial load in rats. The data are shown as the means $\pm \mathrm{SD}$. **, $\mathrm{P}<0.01$ indicates significant differences compared to the control group; ${ }^{*} \mathrm{P}<0.05$ vs. the model group; ${ }^{\#}, \mathrm{P}<0.01$ vs. the model group; ${ }^{\Delta}, \mathrm{P}<0.05$ vs. the $\mathrm{CMZ}$ group; ${ }^{\Delta \Delta}, \mathrm{P}<0.01$ vs. the CMZ group. (B) Changes in the chest $\mathrm{X}$-rays of the rats with severe pneumonia in each group. (C) The effects of RDN and CMZ on histopathological changes in lung tissues in rats with severe pneumonia [hematoxylin-eosin staining (HE), ×200]. RDN, Reduning; CMZ, Cefmetazole. 

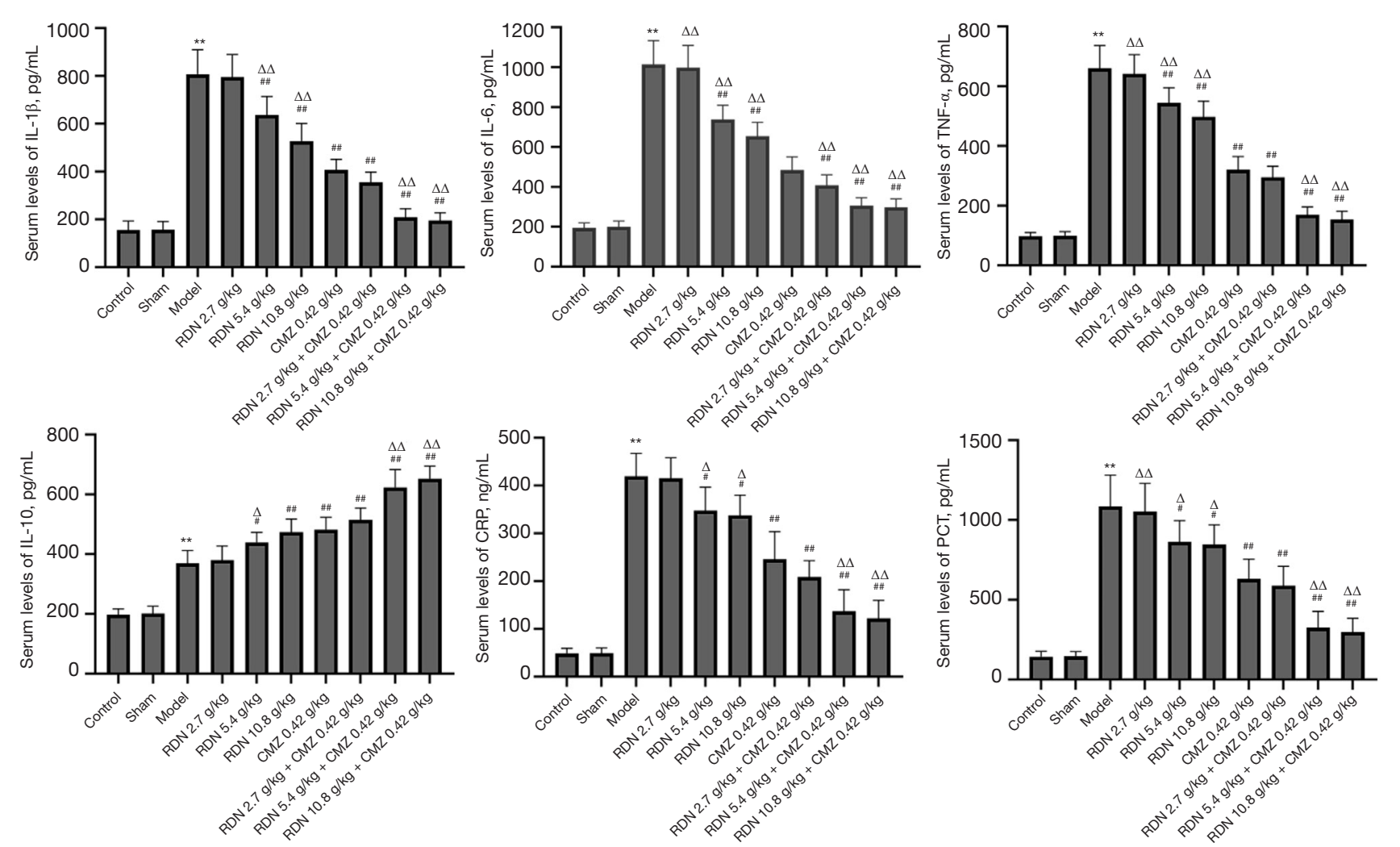

Figure 6 Effects of RDN on inflammatory cytokine (i.e., IL-1 $\beta$, IL-6, TNF- $\alpha$, and IL-10) and inflammation indicator (i.e., CRP and PCT) production in rats with severe pneumonia. The values represent the mean \pm SD of 6 independent experiments, and the differences between the mean values were assessed by the Student's $t$-test. **, $\mathrm{P}<0.01$ vs. the control group; ${ }^{*}, \mathrm{P}<0.05,{ }^{\#}, \mathrm{P}<0.01$ vs. the model group; ${ }^{\Delta}$, $\mathrm{P}<0.05$, ${ }^{\Delta}, \mathrm{P}<0.01$ vs. the CMZ group. IL-6, interleukin 6; TNF- $\alpha$, Tumor Necrosis Factor $\alpha$; IL-10, interleukin 10; IL-1 $\beta$, interleukin 1 $\beta$; CRP, C-reactive protein; PCT, Procalcitonin.

treatment of RDN with CMZ (see Figure $8 A$ ). The same results were obtained by Western blotting (see Figure $8 B$ ). These results indicated that RDN inhibited the activation of M1 macrophages and promoted the polarization of M2 macrophages in rats with severe pneumonia.

\section{Discussion}

Severe pneumonia is a special type of sepsis. When inflammation is not controlled in the early stage of an infection, multiple organs can fail, endangering the life of the patient (20). In recent years, treatments integrating TCM and Western medicine have received increasing attention, and their combined efficacy has been confirmed $(15,20,21)$. During the outbreak of COVID-19, RDN was recommended as a TCM preparation for the clinical treatment of patients with severe COVID-19, and it reduced the inflammatory response and led to positive therapeutic effects (18). This effect was attributed to the anti-inflammatory effects of the active components of RDN. Thus, we hypothesized that RDN may help reduce the inflammatory response in severe pneumonia. In this study, we explored the mechanism of RDN synergism in the treatment of severe pneumonia models in vitro and in vivo.

AhR is a highly conserved nuclear receptor. After binding with ligands, $\mathrm{AhR}$ enters the nucleus to regulate the expression of target genes and is thought to play an important role in regulating immune and inflammatory responses (22). AhR activation can downregulate the expression of TNF- $\alpha$, IL-1 $\beta$, IL- 6 , and other proinflammatory factors, thereby affecting the inflammatory response and immune homeostasis (23). Additionally, studies 
have shown that $A b R$ is essential for the survival of mice in septic shock and regulates systemic inflammation, while the expression levels of the cytokines of IL- $1 \beta$, TNF- $\alpha$, and IL-6 in $A b R^{-/-}$mice are significantly increased after an LPS attack, and the survival rate is decreased $(24,25)$. $A b R$ agonists have also been shown to protect wild-type $A b R$ mice, but not to protect $A b R^{-/-}$mice from septic shock (25). Thus, we believe that $A b R$ may be an important target in sepsis treatment, and drugs acting on $A b R$ targets may represent a new direction for sepsis treatment in the future.

We performed confocal laser scanning microscopy, and found that AhR was activated and entered the nucleus of A549 cells after LPS stimulation, and the expression of AhR in the nucleus was significantly increased after RDN administration. Additionally, the expression of AhR was significantly. more increased when RDN was combined with antibiotics than when RDN or an antibiotic was used alone. RDN may be an activator of AhR, and its effect may be mediated by the increased expression of AhR in the nucleus; this mechanism is similar to that by which activated AhR was previously shown to alleviate colitis in mice (26). In the present study, RDN effectively downregulated the expression levels of TNF- $\alpha$, IL-1 $\beta$, and IL- 6 in LPSstimulated A549 cells, and AhR expression in the cytoplasm of A549 cells was increased, suggesting that AhR played an important role in the control of inflammation. These findings are consistent with the transcriptional expression of Mt-p (a methylated derivative of pelargonidin), which
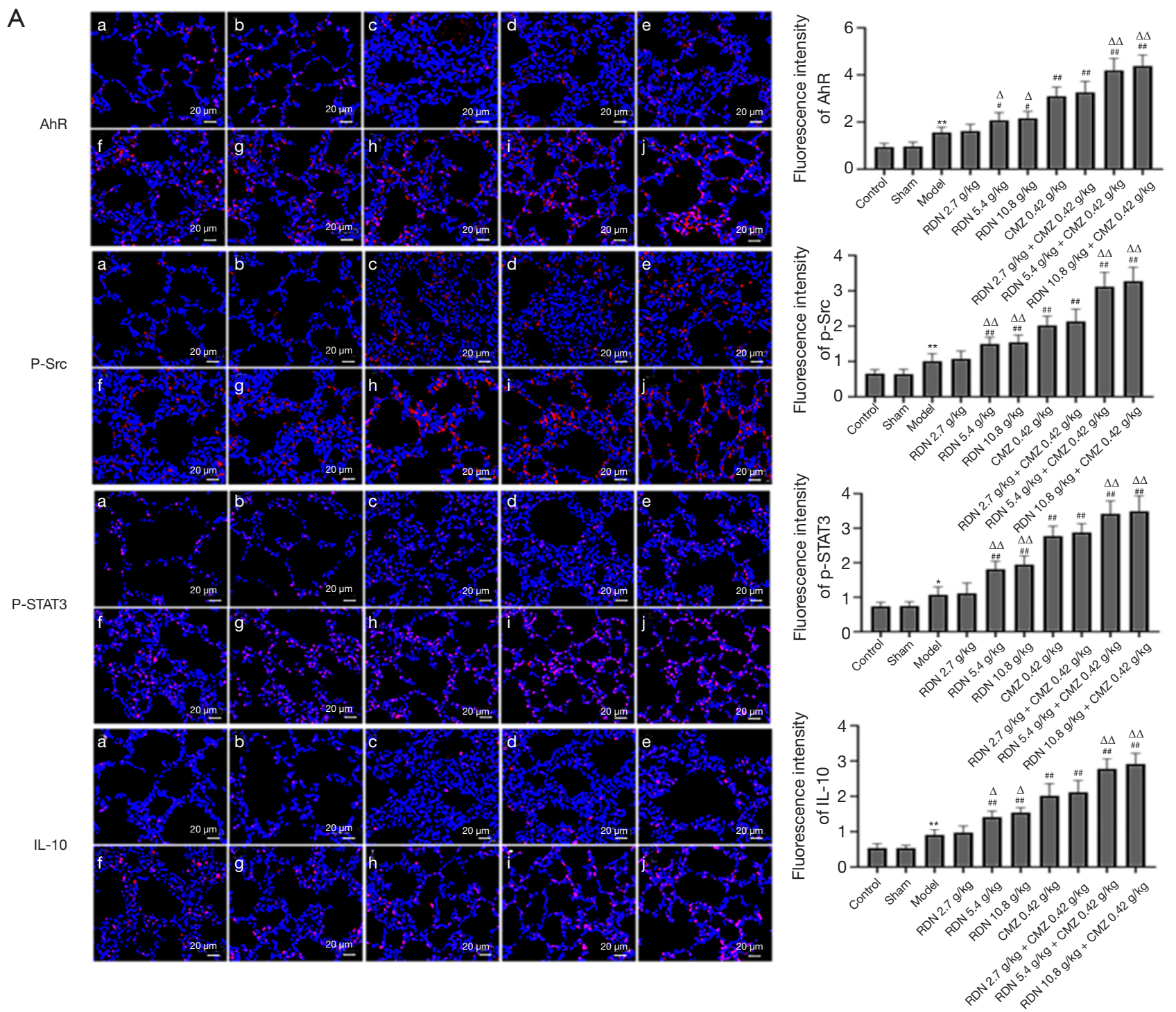

() Journal of Thoracic Disease. All rights reserved. 
B

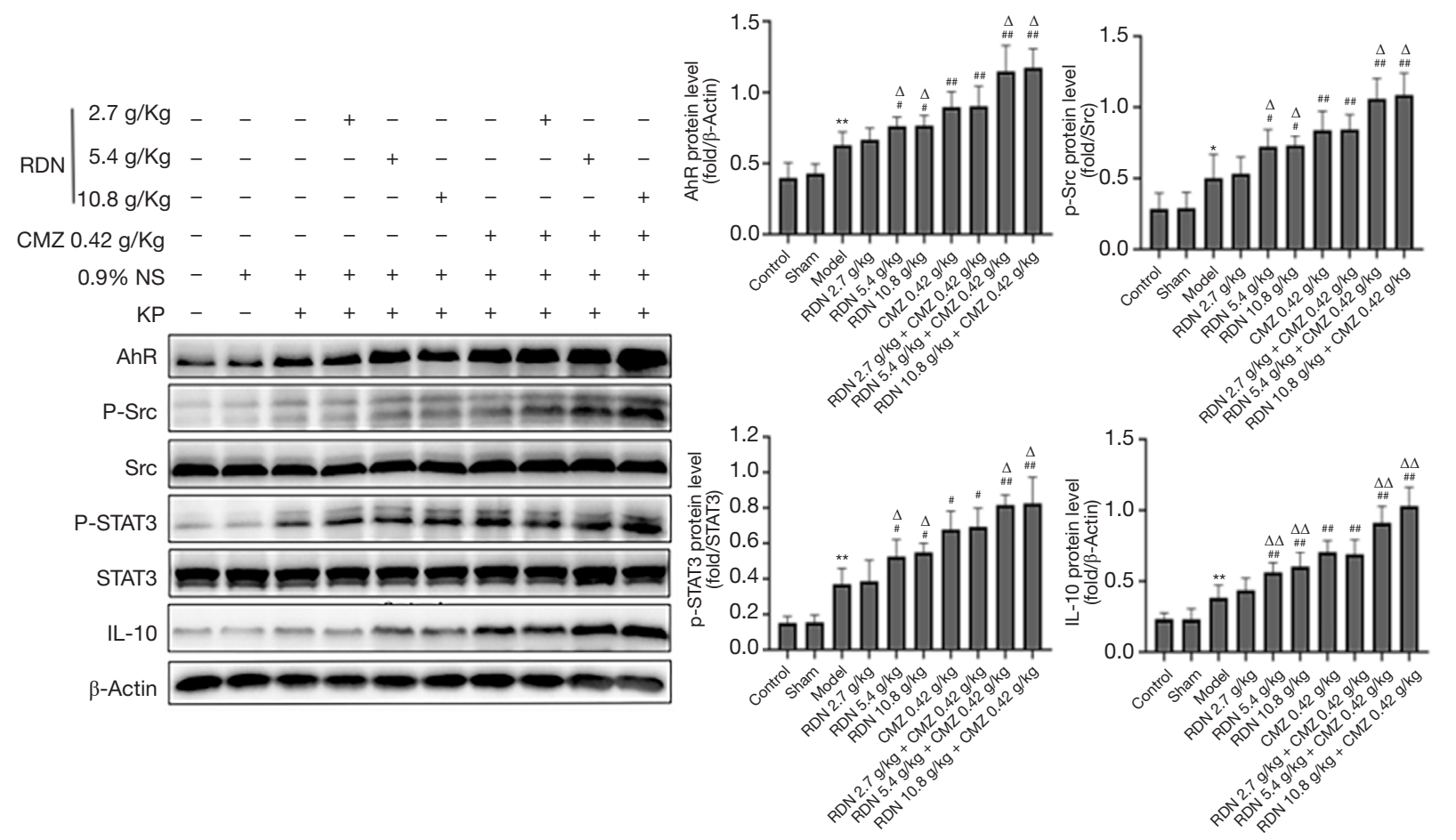

Figure 7 Effects of RDN on the protein expressions of AhR, p-src, p-STAT3 and IL-10 in severe pneumonia rats. (A) Immunofluorescence staining of AhR, p-src, p-STAT3, and IL-10 in the lung tissues of rats with severe pneumonia (red indicates the target protein, $\times 400$ ). (B) The effects of the AhR-Src-STAT3-IL-10 pathway in rats with severe pneumonia. Western blots showing AhR, p-Src, Src, p-STAT3, STAT3, IL-10, and $\beta$-actin expression. The values represent the mean \pm SD of 6 independent experiments, and the differences between the mean values were assessed by the Student's t-test. ${ }^{*}, \mathrm{P}<0.05$, ${ }^{* *}, \mathrm{P}<0.01$ indicates significant differences compared to the control group; \#, $\mathrm{P}<0.05$ vs. the model group; ${ }^{\#}, \mathrm{P}<0.01$ vs. the model; ${ }^{\Delta}, \mathrm{P}<0.05$ vs. the $\mathrm{CMZ}$ group; ${ }^{\Delta \Delta}, \mathrm{P}<0.01$ vs. the CMZ group. RDN, Reduning; CMZ, Cefmetazole; AhR, Aryl hydrocarbon receptor; p-Src, Phosphorylation Src tyrosine kinase; Src, Src tyrosine kinase; p-STAT3, Phosphorylation signal transducer and activator of transcription 3; STAT3, signal transducer and activator of transcription 3; IL-10, interleukin 10 .

a previous in vitro experiment showed activated $\mathrm{AhR}$ and suppressed TNF- $\alpha$, IL-1 $\beta$, and IL- 6 expression (27). Further, in our study, TNF- $\alpha$, IL-1 $\beta$, and IL-6 expression in $\mathrm{AhR}^{-/-} \mathrm{A} 549$ cells was significantly increased after LPS stimulation. These results indicated that AhR is an important target for controlling inflammation, and that $\mathrm{RDN}$ is its ligand.

Studies have shown that Src is the downstream target of AhR, which can be activated by AhR and then phosphorylated to exert its physiological effects $(28,29)$. STAT3 plays a key role in controlling inflammation and modulating immunity. STAT3 activates the Src-mediated IL-10 pathway, an important intracellular inflammatory signal transduction pathway (30). We found a protein interaction between AhR and STAT3 through a Co-IP experiment. After A549 inflammatory cells were treated with RDN, AhR entry into the nucleus was significantly increased, Src and STAT3 were activated, and their phosphorylation expression was significantly increased, and thus, the expression of IL-10 was upregulated. In the A549-THP-1 coculture system, RDN increased the expression of p-Src and p-STAT3 through AhR activation of the Src-STAT3 axis, thus targeting the expression of IL-10 and playing an anti-inflammatory role. Increased STAT3 phosphorylation and subsequently increased IL-10 expression play an anti-inflammatory role similar to the protective effect observed in ulcerative colitis (31).

To further investigate the effect of RDN on the AhRSrc-STAT3-IL-10 pathway, we transfected A549 cells with lentivirus to silence the $A b R$ gene. The results 
A
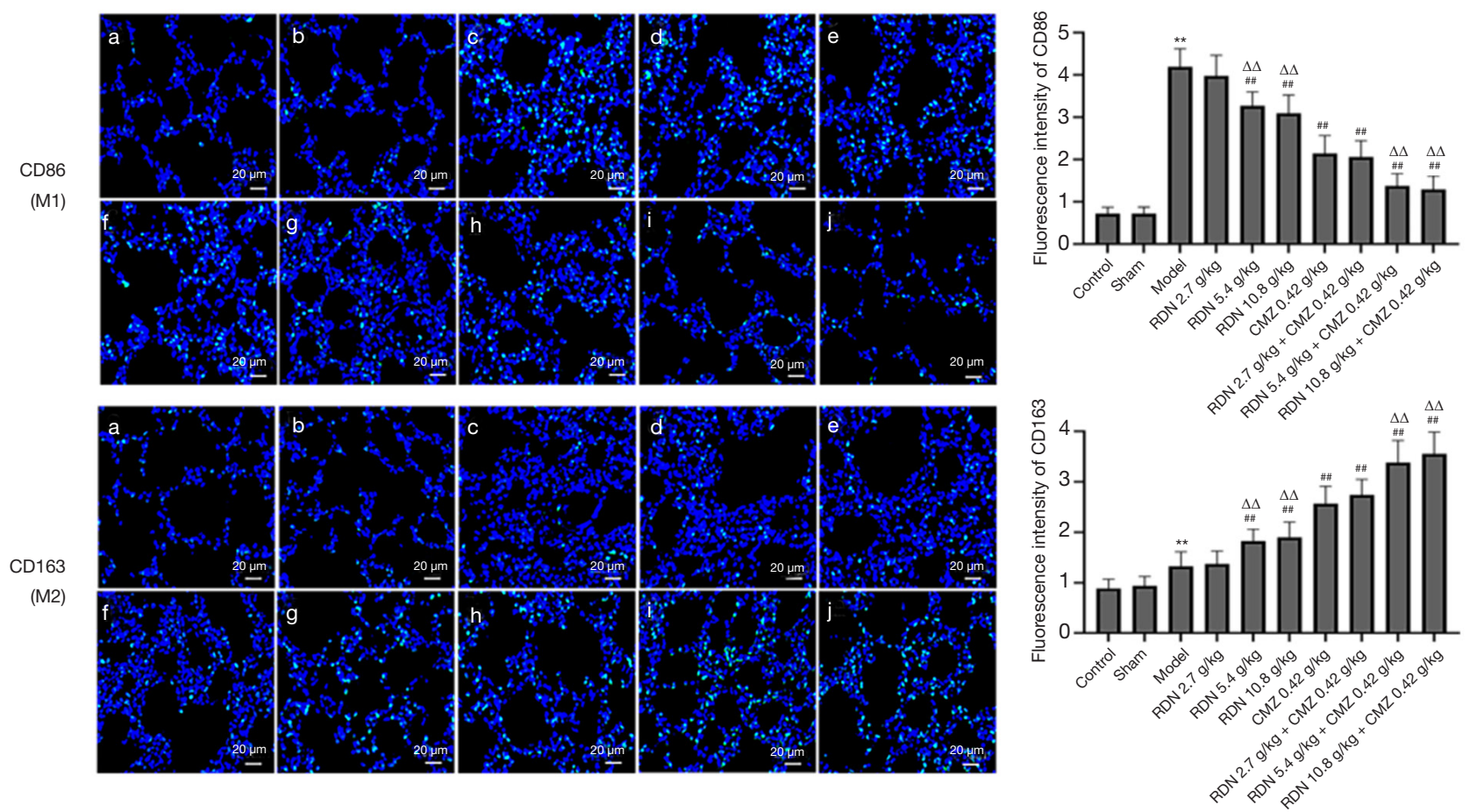

B
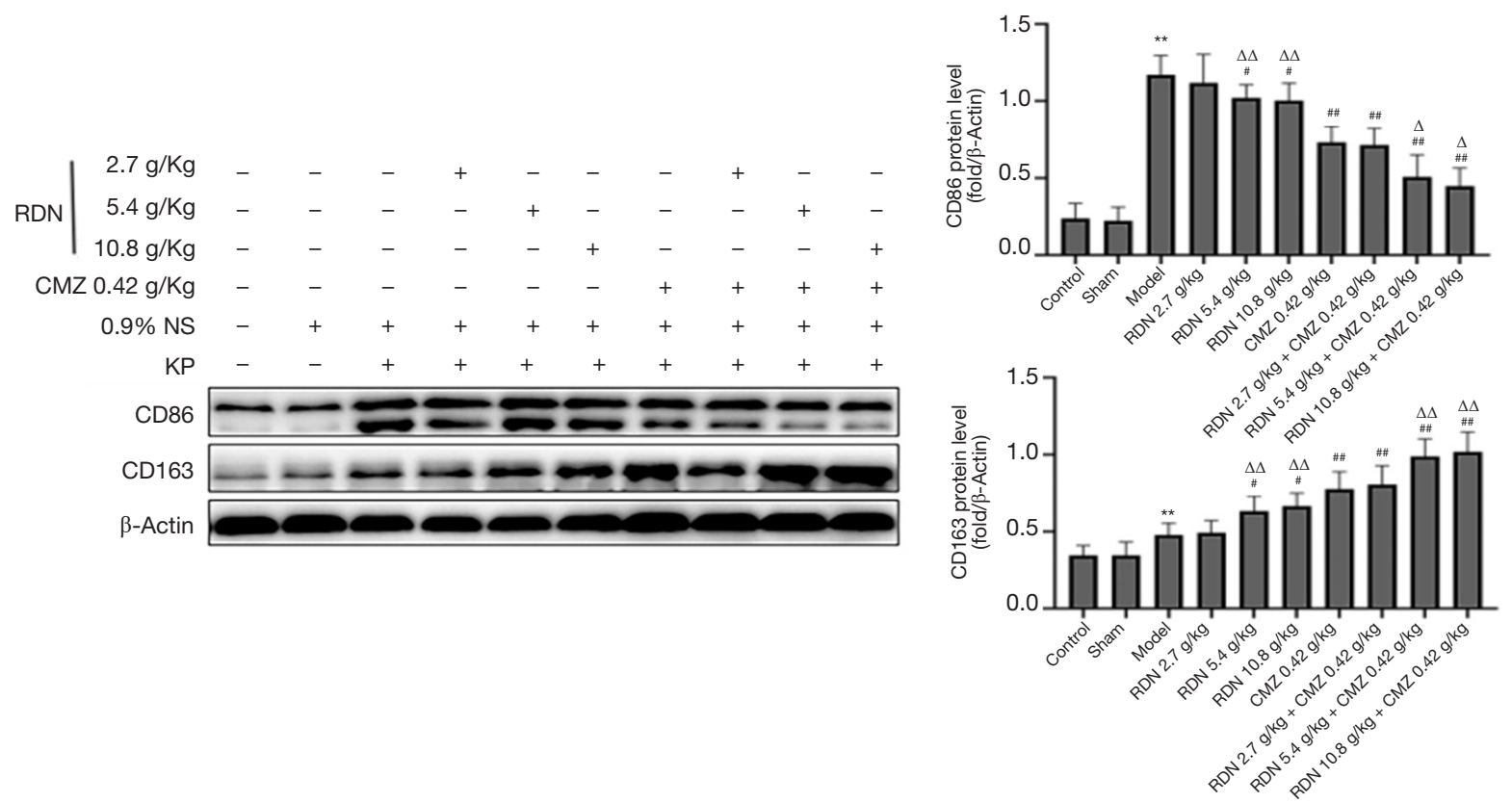

Figure 8 Effects of RDN on the protein expressions of CD86 and CD163 in severe pneumonia rats. (A) Immunofluorescence staining of the lung tissues of the rats with severe pneumonia (green indicates the target protein, $\times 400$ ). (B) Western blots showing CD86, CD163, and $\beta$-actin. The values represent the mean $\pm \mathrm{SD}$ of 6 independent experiments, and the differences between mean values were assessed by the Student's $t$-test. ${ }^{* *}, \mathrm{P}<0.01$ vs. the control group; ${ }^{\prime}, \mathrm{P}<0.05,{ }^{\prime \prime \prime}, \mathrm{P}<0.01$ vs. the model group; ${ }^{\Delta}, \mathrm{P}<0.05,{ }^{\Delta \Lambda}, \mathrm{P}<0.01$ vs. the CMZ group. RDN, Reduning; CMZ, cefmetazole; CD86, cluster of differentiation 86; CD163, cluster of differentiation 163. 
showed that Src and STAT3 phosphorylation and IL-10 expression decreased significantly after $A b R$ silencing but increased slightly after RDN treatment, but no significant difference was observed. The expression of TNF- $\alpha$, IL- $1 \beta$ and IL- 6 was significantly increased in the inflammatory cell model after $A b R$ silencing but was decreased in the inflammatory cell model after the RDN intervention, but no significant difference was detected. These results suggest that the Src-STAT3-IL-10 pathway activated by AhR may be one of the main mechanisms by which RDN exerts anti-inflammatory effects. We also observed that the expression of proinflammatory factors in $A b R$ silenced A549 cells decreased to a certain extent after RDN treatment, which indicates that other mechanisms play an anti-inflammatory role. In future studies, we will continue to explore other possible mechanisms of action. Compared with the RDN alone treatment, the treatment of RDN combined with CMZ significantly increased Src, STAT3 phosphorylation, and IL-10 expression, reduced proinflammatory factor expression, alleviated lung injury in rats with severe pneumonia, and improved the survival rate of the rats. Additionally, RDN has also been proven to exert a good protective effect on paraquat- and LPS-induced acute lung injury in rats $(32,33)$. In summary, RDN has a therapeutic effect on severe pneumonia and can increase the effect of CMZ.

The imbalance between proinflammatory and antiinflammatory pathways in the host is key to the development of sepsis (34). M1 macrophages secrete proinflammatory cytokines, and M2 macrophages secrete anti-inflammatory cytokines (35). Macrophages are the key inflammatory cells in the early stage of severe pneumonia. When M1 macrophages are overactivated, the proinflammatory effect is significantly stronger than the anti-inflammatory effect, and thus aggravate the pulmonary inflammatory response (36). The activation of AhR can promote the transformation of M1 macrophages into M2 macrophages (10). We found that RDN also promoted the polarization of macrophages toward the anti-inflammatory M2 phenotype by inhibiting the polarization of macrophages toward the M1 phenotype. Additionally, RDN combined with antibiotics was also observed to have an effect on macrophage polarization. RDN combined with antibiotics significantly reduced M1 polarization and promoted M2 polarization to a greater extent than the effect of RDN or antibiotics alone. Regarding the potential mechanism by which this combination treatment inhibits M1 polarization and promotes $\mathrm{M} 2$ polarization, it has been previously found that the downregulation of the nuclear factor kappa-B(NF- $\kappa \mathrm{B})$ signaling pathway can inhibit M1 polarization, while the activation of STAT3 can promote M2 polarization. These results are similar to our results (37). As mentioned above, STAT3 activation by AhR was observed in our experiment, and the degree of M2 macrophage differentiation increased under the RDN intervention. Thus, STAT3 activation by $\mathrm{AhR}$ may be the mechanism by which RDN regulates macrophage polarization.

In conclusion, this study suggests that RDN has a strong anti-inflammatory effect. RDN combined with CMZ in the treatment of severe pneumonia can significantly reduce inflammatory factors, reduces lung injury, improves the survival rate, and shorten the course of disease than antibiotics alone, thereby reducing the use of antibiotics and the generation of drug-resistant bacteria. The mechanism may involve Src-STAT3-IL-10 pathway activation by AhR, which inhibits M1 polarization and promotes M2 polarization of macrophages, thereby reducing the expression of proinflammatory factors and increasing the expression of anti-inflammatory factors. This conclusion supports the use of RDN in the treatment of early severe pneumonia; however, further clinical studies still need to be conducted to prove its effectiveness in clinical settings.

\section{Acknowledgments}

Funding: This study was funded by the National Natural Science Foundation of China (Nos. 81871611, 81760674, and 82060678). No study sponsors were involved in the research process of this project.

\section{Footnote}

Reporting Checklist: The authors have completed the ARRIVE reporting checklist. Available at https://jtd. amegroups.com/article/view/10.21037/jtd-22-126/rc

Data Sharing Statement: Available at https://jtd.amegroups. com/article/view/10.21037/jtd-22-126/dss

Conflicts of Interest: All authors have completed the ICMJE uniform disclosure form (available at https://jtd.amegroups. com/article/view/10.21037/jtd-22-126/coif). The authors have no conflicts of interest to declare.

Ethical Statement: The authors are accountable for all aspects of the work in ensuring that questions related to the accuracy or integrity of any part of the work are 
appropriately investigated and resolved. All experiments were reviewed and approved by the Institutional Animal Care and Treatment Committee of Hainan Medical University (No. HYLL-2021-159), in compliance with Guidelines for the Humane Treatment of Laboratory Animals (Ministry of Science and Technology of the People's Republic of China; policy No. 2006398).

Open Access Statement: This is an Open Access article distributed in accordance with the Creative Commons Attribution-NonCommercial-NoDerivs 4.0 International License (CC BY-NC-ND 4.0), which permits the noncommercial replication and distribution of the article with the strict proviso that no changes or edits are made and the original work is properly cited (including links to both the formal publication through the relevant DOI and the license). See: https://creativecommons.org/licenses/by-nc-nd/4.0/.

\section{References}

1. Leoni D, Rello J. Severe community-acquired pneumonia: optimal management. Curr Opin Infect Dis 2017;30:240-7.

2. Sligl WI, Marrie TJ. Severe community-acquired pneumonia. Crit Care Clin 2013;29:563-601.

3. Kiedrowski MR, Bomberger JM. Viral-Bacterial Coinfections in the Cystic Fibrosis Respiratory Tract. Front Immunol 2018;9:3067.

4. Torres A, Chalmers JD, Dela Cruz CS, et al. Challenges in severe community-acquired pneumonia: a point-of-view review. Intensive Care Med 2019;45:159-71.

5. Phua J, Dean NC, Guo Q, et al. Severe communityacquired pneumonia: timely management measures in the first 24 hours. Crit Care 2016;20:237.

6. Wongsurakiat P, Chitwarakorn N. Severe communityacquired pneumonia in general medical wards: outcomes and impact of initial antibiotic selection. BMC Pulm Med 2019;19:179.

7. Dodds DR. Antibiotic resistance: A current epilogue. Biochem Pharmacol 2017;134:139-46.

8. Garnacho-Montero J, Barrero-García I, Gómez-Prieto MG, et al. Severe community-acquired pneumonia: current management and future therapeutic alternatives. Expert Rev Anti Infect Ther 2018;16:667-77.

9. Bock KW. Aryl hydrocarbon receptor (AHR) functions: Balancing opposing processes including inflammatory reactions. Biochem Pharmacol 2020;178:114093.

10. Lv C, Huang L. Xenobiotic receptors in mediating the effect of sepsis on drug metabolism. Acta Pharm Sin B
2020;10:33-41.

11. Hillmer EJ, Zhang H, Li HS, et al. STAT3 signaling in immunity. Cytokine Growth Factor Rev 2016;31:1-15.

12. Bessede A, Gargaro M, Pallotta MT, et al. Aryl hydrocarbon receptor control of a disease tolerance defence pathway. Nature 2014;511:184-90.

13. Yang $X$, Liu H, Ye T, et al. AhR activation attenuates calcium oxalate nephrocalcinosis by diminishing M1 macrophage polarization and promoting M2 macrophage polarization. Theranostics 2020;10:12011-25.

14. Wang Y, Liang $H$, Jin F, et al. Injured liver-released miRNA-122 elicits acute pulmonary inflammation via activating alveolar macrophage TLR7 signaling pathway. Proc Natl Acad Sci U S A 2019;116:6162-71.

15. Cao C, Zhen Z, Kuang S, et al. Reduning injection combined with western medicine for pneumonia: A protocol for systematic review and meta-analysis. Medicine (Baltimore) 2020;99:e22757.

16. Liu J, Sun K, Zheng C, et al. Pathway as a pharmacological target for herbal medicines: an investigation from reduning injection. PLoS One 2015;10:e0123109.

17. Li Y, Wang P, Xiao W, et al. Screening and analyzing the potential bioactive components from reduning injection, using macrophage cell extraction and ultra-high performance liquid chromatography coupled with mass spectrometry. Am J Chin Med 2013;41:221-9.

18. Xu X, Zhang J, Zheng W, et al. Efficacy and safety of Reduning injection in the treatment of COVID-19: a randomized, multicenter clinical study. Ann Palliat Med 2021;10:5146-55.

19. Chen W, Ma Y, Zhang H, et al. Reduning plus ribavirin display synergistic activity against severe pneumonia induced by $\mathrm{H} 1 \mathrm{~N} 1$ influenza A virus in mice. J Tradit Chin Med 2020;40:803-11.

20. Hermann S, Brandes F, Kirchner B, et al. Diagnostic potential of circulating cell-free microRNAs for community-acquired pneumonia and pneumonia-related sepsis. J Cell Mol Med 2020;24:12054-64.

21. Liu LW, Shi YY, Li ZL, et al. Metabolomic Insights Into the Synergistic Effect of Biapenem in Combination With Xuebijing Injection Against Sepsis. Front Pharmacol 2020;11:502.

22. Neavin DR, Liu D, Ray B, et al. The Role of the Aryl Hydrocarbon Receptor (AHR) in Immune and Inflammatory Diseases. Int J Mol Sci 2018;19:3851.

23. Zhu C, Xie Q, Zhao B. The role of AhR in autoimmune regulation and its potential as a therapeutic target against CD4 $\mathrm{T}$ cell mediated inflammatory disorder. Int J Mol Sci 
2014;15:10116-35.

24. Sekine H, Mimura J, Oshima M, et al. Hypersensitivity of aryl hydrocarbon receptor-deficient mice to lipopolysaccharide-induced septic shock. Mol Cell Biol 2009;29:6391-400.

25. Huai $W$, Zhao R, Song H, et al. Aryl hydrocarbon receptor negatively regulates NLRP3 inflammasome activity by inhibiting NLRP3 transcription. Nat Commun 2014;5:4738.

26. Lv Q, Shi C, Qiao S, et al. Alpinetin exerts anticolitis efficacy by activating AhR, regulating miR-302/ DNMT-1/CREB signals, and therefore promoting Treg differentiation. Cell Death Dis 2018;9:890.

27. Biagioli M, Carino A, Fiorucci C, et al. The Aryl Hydrocarbon Receptor (AhR) Mediates the CounterRegulatory Effects of Pelargonidins in Models of Inflammation and Metabolic Dysfunctions. Nutrients 2019;11:1820.

28. Solis NV, Swidergall M, Bruno VM, et al. The Aryl Hydrocarbon Receptor Governs Epithelial Cell Invasion during Oropharyngeal Candidiasis. mBio 2017;8:00025-17.

29. Assefa EG, Yan Q, Gezahegn SB, et al. Role of Resveratrol on Indoxyl Sulfate-Induced Endothelial Hyperpermeability via Aryl Hydrocarbon Receptor (AHR)/Src-Dependent Pathway. Oxid Med Cell Longev 2019;2019:5847040.

30. Zhu J, Luo L, Tian L, et al. Aryl Hydrocarbon Receptor Promotes IL-10 Expression in Inflammatory Macrophages
Through Src-STAT3 Signaling Pathway. Front Immunol 2018;9:2033.

31. Wang G, Xu B, Shi F, et al. Protective Effect of MethaneRich Saline on Acetic Acid-Induced Ulcerative Colitis via Blocking the TLR4/NF-кB/MAPK Pathway and Promoting IL-10/JAK1/STAT3-Mediated Antiinflammatory Response. Oxid Med Cell Longev 2019;2019:7850324.

32. Jiang C, Zhong R, Zhang J, et al. Reduning injection ameliorates paraquat-induced acute lung injury by regulating AMPK/MAPK/NF- $\kappa$ B signaling. J Cell Biochem 2019;120:12713-23.

33. Tang LP, Xiao W, Li YF, et al. Anti-inflammatory effects of Reduning Injection on lipopolysaccharide-induced acute lung injury of rats. Chin J Integr Med 2014;20:591-9.

34. Venet F, Monneret G. Advances in the understanding and treatment of sepsis-induced immunosuppression. Nat Rev Nephrol 2018;14:121-37.

35. Mills CD. Anatomy of a discovery: $\mathrm{m} 1$ and $\mathrm{m} 2$ macrophages. Front Immunol 2015;6:212.

36. Mizgerd JP. Pathogenesis of severe pneumonia: advances and knowledge gaps. Curr Opin Pulm Med 2017;23:193-7.

37. Yang X, Xu S, Qian Y, et al. Resveratrol regulates microglia $M 1 / M 2$ polarization via PGC-1 $\alpha$ in conditions of neuroinflammatory injury. Brain Behav Immun 2017;64:162-72.
Cite this article as: Luo S, Gan L, Liu S, Zhong L, Chen M, Zhang H, Li J, Huang L, Lv C. The synergistic Reduning and cefmetazole sodium treatment of severe pneumonia is mediated by the AhR-Src-STAT3 pathway. J Thorac Dis 2022;14(2):474493. doi: $10.21037 /$ jtd-22-126 